\title{
Simulação perfeita da distribuição normal multivariada truncada
}

\author{
THIAGO FEITOSA CAMPOS
}

\author{
DisSERTAÇÃO APRESENTADA \\ $\mathrm{AO}$ \\ Instituto de Matemática e Estatística \\ DA \\ Universidade DE SÃo PaUlo \\ PARA \\ OBTENÇÃO DO TÍTULO \\ $\mathrm{DE}$ \\ Mestre em CiênCIAS
}

Programa: Estatística

Orientador: Profa. Dra. Márcia D’Elia Branco

Durante o desenvolvimento deste trabalho o autor recebeu auxílio financeiro da CAPES/CNPq

São Paulo, maio de 2010 


\title{
Simulação perfeita da distribuição normal multivariada truncada
}

\author{
Este exemplar corresponde à redação \\ final da dissertação devidamente corrigida \\ e defendida por Thiago Feitosa Campos \\ e aprovada pela Comissão Julgadora.
}

Banca Examinadora:

- Profa. Dra. Márcia D’Elia Branco (orientadora) - IME-USP.

- Prof. Dr. Fabio Prates Machado - IME-USP.

- Profa. Dra. Nancy Lopes Garcia - UNICAMP. 


\section{Agradecimentos}

Quero agradecer em primeiro lugar a Deus por ter proporcionado toda a minha vida até agora. Aos meus pais por terem me criado e me educado o melhor que podiam. Agradecer também aos meus tios Cleiton e Acácio por terem apoiado minha educação.

Agradeço também aos meus professores, desde a educação infantil até a graduação na UFC, pois sem o empenho, esforço deles eu não teria chegado tão longe. Em especial, agradeço aos professores André Jalles Monteiro, João Maurício Araújo Mota e Juvêncio Santos Nobre pelo incentivo a pesquisa científica.

Agradeço aos meus amigos Daniel "Kblo", Emanuel Carvalho, Raymond Sideaux, Jairo "Senpai", Marcelo Gadelha, Eduardo "Monstrin", Gabriella Serra, Gabriela Cabral, Priscilla Barbosa, Elisa Strobel por terem me apoiado e acreditado em mim durante a minha graduação e o período anterior a prova de admissão do mestrado. Quero também agradecer ao Alexandre e Fabio, amigos de republica, pelas horas de descontração e pro terem me ajudado a crescer. Ao João Italo pelas ajudas com os algoritmos. Aos Amigos do IME pelo apoio nas horas difíceis nas disciplinas. Ao amigos do Karate por ajudarem a aliviar todo o stress que o mestrado me deu. em especial ao Caio Gustavo por me aturar tanto tempo e a Amanda Leal pelas revisões de algumas partes da dissertação e a tradução do resumo para a língua inglesa.

Quero também agradecer a professora Márcia por ter me orientado, e aos professores Fabio e Nancy por estarem na minha banca e terem lido meu trabalho e ajudado a melhora-lo.

Quero agradecer também as instituições federais que me deram apoio financeiro durante esse trabalho, a CAPES e o CNPq. 


\section{Resumo}

No presente trabalho apresentamos o algoritmo de simulação perfeita CFTP, proposto em Propp \& Wilson (1996). Seguindo o trabalho de Philippe \& Robert (2003) implementamos o CFTP gerando amostras da distribuição normal bivariada truncada no quadrante positivo. $\mathrm{O}$ algoritmo proposto é comparado com o amostrador de Gibbs e o método de rejeição. Finalmente, apresentamos sugestões para a implementação do CFTP para gerar amostras da distribuição normal truncada em dimensões maiores que dois e a geração de amostras em conjuntos diferente do quadrante positivo. Palavras-chave: CFTP, distribuição multivariada truncada, amostrador perfeito. 


\section{Abstract}

This Project will display the CFTP perfect simulation algorithm presented at Propp \& Wilson (1996). According to Philippe \& Robert (2003) will be implemented the CFTP providing samples of the bivariate normal distribution truncated at the positive quadrant. The proposed algorithm is compared to the samples generated by Gibbs Sampler and by the rejection sampling ( or acceptancerejection method or "accept-reject algorithm"). Finally, suggestions to the implementation of CFTP in order to produce truncated normal distribution samples at bigger dimensions than two and the provide a different set of samples from the positive quadrant Keywords: CFTP, truncated multivariate distribution, perfect sampler. 


\section{Sumário}

Listas de abreviaturas e símbolos $\quad$ v

Lista de Figuras $\quad$ vi

Lista de Tabelas $\quad$ vii

1 Introdução $\quad 1$

1.1 Organização do Trabalho . . . . . . . . . . . . . . . . . . 1

2 Algoritmo CFTP $\quad 3$

2.1 Definições e propriedades do algoritmo CFTP . . . . . . . . . . . . . . . . 3

2.1 .1 Acoplamento . . . . . . . . . . . . . . . . 4

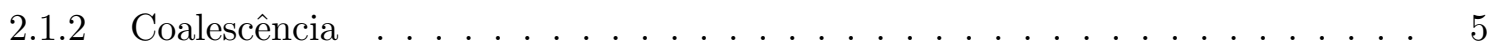

2.2 Implementação do CFTP . . . . . . . . . . . . . . . . . . . . . . . . 5

2.3 CFTP monótono . . . . . . . . . . . . . . . . . . 7

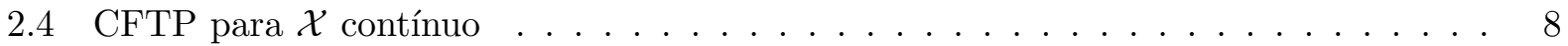

3 Simulação da normal bivariada truncada $\quad 12$

3.1 Amostrador perfeito . . . . . . . . . . . . . . . . . . . 13

3.1 .1 Evolução das cadeias de Markov e função de transição . . . . . . . . . . . . . . 14

4 Análise dos dados simulados $\quad 16$

4.1 Análise gráfica . . . . . . . . . . . . . . . . . . . . 17

4.1 .1 Análise marginal . . . . . . . . . . . . . . . . 17

4.2 Análise do tempo computacional . . . . . . . . . . . . . . . . . . 17

4.2.1 Influência da correlação no tempo computacional do CFTP . . . . . . . . . . 18

4.2 .2 Considerações finais . . . . . . . . . . . . . . . . . 20

$\begin{array}{ll}\text { A Algoritmo } & 21\end{array}$

$\begin{array}{ll}\text { B Gráficos } & 24\end{array}$

$\begin{array}{ll}\text { Referências Bibliográficas } & 29\end{array}$ 


\title{
Listas de abreviaturas e símbolos
}

\author{
CFTP Acoplamento do Passado (Coupling From The Past). \\ MCMC Monte Carlo Cadeia de Markov (Monte Carlo Markov Chain) \\ CM Cadeia de Markov \\ $\pi \quad$ Distribuição estacionária de uma cadeia de Markov. \\ $\phi \quad$ Função de transição de uma cadeia de Markov \\ $\mathcal{X} \quad$ Espaço de estados
}




\section{Lista de Figuras}

2.1 Trajetórias de cadeias de Markov atualizadas para $T=-1 \ldots \ldots \ldots$

2.2 Trajetórias de cadeias de Markov atualizadas para $T=-2 \ldots \ldots \ldots$

2.3 Trajetórias de cadeias de Markov atualizadas para $T=-3 \ldots \ldots \ldots$

3.1 Caixa e elipsoide gerados pelo algoritmo de aceitação e rejeição . . . . . . . . . . 15

B.1 Amostras da distribuição normal bivariada truncada no quadrante positivo no caso 1, respectivamente, geradas pelos três algoritmos, com a sobreposição de HPDs de $50 \%$ de probabilidade acumulada, $80 \%, 85 \%, 90 \%, 95 \%$ e $99 \%$. . . . . . . 24

B.2 Amostras da distribuição normal bivariada truncada no quadrante positivo no caso 2, geradas pelos três algoritmos, com a sobreposição de HPDs de $50 \%$ de probabilidade acumulada, $80 \%, 85 \%, 90 \%, 95 \%$ e $99 \% \ldots \ldots \ldots$

B.3 Amostras da distribuição normal bivariada truncada no quadrante positivo no caso 3 , respectivamente, geradas pelos três algoritmos, com a sobreposição de HPDs de $50 \%$ de probabilidade acumulada, $80 \%, 85 \%, 90 \%, 95 \%$ e $99 \%$. . . . . . . 25

B.4 Amostras da distribuição normal bivariada truncada no quadrante positivo no caso 4, respectivamente, geradas pelos três algoritmos, com a sobreposição de HPDs de $50 \%$ de probabilidade acumulada, $80 \%, 85 \%, 90 \%, 95 \%$ e $99 \%$. . . . . . . 26

B.5 Histograma das densidades marginais estimadas, com sobreposição da densidade de interesse, para os dados simulados no primeiro caso por cada um dos métodos usados 26

B.6 Histograma das densidades marginais estimadas, com sobreposição da densidade de interesse, para os dados simulados no segundo caso por cada um dos métodos usados 27

B.7 Histograma das densidades marginais estimadas, com sobreposição da densidade de interesse, para os dados simulados no terceiro caso por cada um dos métodos usados

B.8 Histograma das densidades marginais estimadas, com sobreposição da densidade de interesse, para os dados simulados no quarto caso por cada um dos métodos usados . 28

B.9 Histograma dos tempos de retornos para que ocorra a coalescência das cadeias de Markov que representam os estados 0 e $\hat{1}$. O gráfico (a) refere as amostras da distribuição com $\mu_{1}$. O gráfico (b) refere as amostras da distribuição com $\mu_{2}$. O gráfico (c) refere as amostras da distribuição com $\mu_{3}$. O gráfico (d) refere as amostras

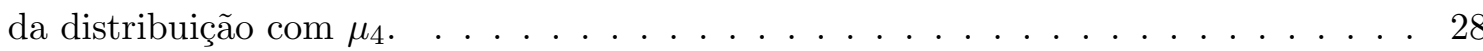




\section{Lista de Tabelas}

4.1 Tempos computacionais, em segundos, gasto pelos três métodos para gerar uma amostra de tamanho $1000 \ldots \ldots \ldots \ldots$

4.2 Tempos computacionais, em segundos, gasto pelo CFTP para gerar amostras com diferentes correlações . . . . . . . . . . . . . . . . . . . . 20 


\section{Capítulo 1}

\section{Introdução}

Em muitos trabalhos estatísticos temos a necessidade de gerar, artificialmente, amostras de distribuições de probabilidade, como por exemplo processamento de sinais, modelos de fatores, modelos tobit multivariado.

Em trabalhos da área de estatística bayesiana o problema de gerar uma amostra de uma distribuição normal multivariada truncada é comum. Um exemplo disso é visto em Farias (2007) onde é necessária a geração de amostras de distribuições normais truncadas multivariadas, e para tal é utilizado o algoritmo de Gibbs. Em Robert (1995) é apresentado uma configuração do algoritmos de Gibbs para gerar amostras da distribuição normal multivariada truncada em um conjunto $\mathcal{A}$ qualquer.

Apresentamos nesse trabalho uma alternativa ao algoritmo de Gibbs, um algoritmo de simulação perfeita, para gerar amostras da distribuição normal multivariada truncada. Mais precisamente a distribuição normal bidimensional truncada no quadrante positivo. Comparamos o algoritmo de simulação perfeita com o amostrador de Gibbs e com o algoritmo de rejeição sem a intenção de determinar qual dos métodos é o melhor. Fernandez, Ferrari \& Grynberg (2007) comentam que o algoritmo de rejeição, em algumas regiões e em duas dimensões, pode ser melhor que o algoritmo de simulação perfeita, mas a situação se inverte quando geramos dados em dimensões maiores que dois. O algoritmo de simulação perfeita fica cada vez melhor quando o número de dimensões aumenta.

\subsection{Organização do Trabalho}

No Capítulo 2 apresentamos o algoritmo de simulação perfeita Coupling From The Past(CFTP), bem como suas propriedades. Descrevemos também a versão mais específica do CFTP, o CFTPmonótono, que é a aplicação do CFTP para quando temos uma ordem natural dos estados e podemos determinar um estado que minimiza a distribuição de interesse, denotado por $\hat{0}$; e um estado que maximiza essa mesma distribuição, denotado por 1̂. Apresentamos também o CFTP quando temos um espaço de estados contínuo, essencial para a geração de amostras em distribuições contínuas.

No Capítulo 3 descrevemos métodos para gerar amostras da distribuição normal bivariada truncada no quadrante positivo. Esse métodos são, o algoritmo de rejeição, o algoritmo de Gibbs e o CFTP. Mostramos a configuração do CFTP para gerar amostras da distribuição normal bivariada truncada no quadrante positivo. 
No Capítulo 4 apresentamos e analisamos os dados simulados da distribuição normal bivariada truncada através do CFTP, amostrador de Gibbs e do algoritmo de rejeição. Analisamos, graficamente, o ajuste dos dados e expomos os tempos computacionais gastos para gerar as amostras.

Em apêndice apresentamos o código fonte do algoritmo desenvolvido para gerar as amostras da distribuição normal bivariada truncada através do CFTP. Comentamos as linhas de comando e sugerimos modificações no algoritmo para gerar amostras em dimensões maiores que dois e com um trucamento em diferentes conjuntos. 


\section{Capítulo 2}

\section{Algoritmo CFTP}

Neste capítulo vamos descrever algumas características importantes do algoritmo de simulação perfeita, denominado Coupling from the past(CFTP) ou acoplamento do passado. Em Propp \& Wilson (1996) foi proposto um algoritmo que tira proveito da coalescência de trajetórias de cadeias de Markov(CM) acopladas, sobre o mesmo espaço de estados. Além disso, o CFTP se baseia na idéia de que se uma CM, $\left\{X_{t}\right\}_{t \in \mathbb{Z}}$, é iniciada no tempo $t=-\infty$ em qualquer estado esteja equilibrada no tempo $t=0$, então o valor da CM no instante zero seria uma amostra da distribuição estacionária de cadeia, nomeada de $\pi$.

\subsection{Definições e propriedades do algoritmo CFTP}

Na presente seção apresentamos algumas definições importantes para o entendimento do algoritmo CFTP. Definições mais precisa das características(irredutibilidade, aperiodicidade, ergodicidade e estacionariedade) de CM podem ser encontradas, por exemplo, no quarto capítulo de Ross (2007). Recordamos alguns conceitos importantes de processos estocásticos para o caso em que o espaço de estados $\mathcal{X}$ é discreto

- Uma CM é irredutivel quando todos os estados pertencentes ao espaço de estados comunicamse entre si.

- Suponha que uma CM iniciada no estado i, e que a cadeia pode retornar ao estado i nos tempos $2,4,6,8, \ldots$, nesse caso o estado i tem período 2 . Um estado é dito aperiódico se o período é igual a 1 . Se todos os estados da cadeia forem aperiódicos então a CM é aperiódica.

- Se um estado i é tal que a probabilidade de, partindo do estado i, retornarmos para o mesmo estado em um numero finito de passos é igual a 1, então o estado i é chamado de recorrente. Uma CM onde todos os estados são recorrentes é chamada de recorrente positiva.

- Uma CM é ergódica se ela é aperiódica e recorrente positiva.

- Uma CM é estacionária se existe um limite da probabilidade de partindo do estado i chegarmos no estado $\mathbf{j}$ em $\mathbf{n}$ passos é independente de $\mathbf{i}$, para qualquer estado $\mathbf{j}$ pertencente ao espaço de estados, denotado por $\lim _{n \rightarrow \infty} P_{i j}^{n}$. 


\subsubsection{Acoplamento}

O acoplamento de duas variáveis aleatórias $X$ e $Y$ é uma variável aleatória $Z=(U, V)$ tal que $U$ tem a mesma distribuição de $X$ e $V$ tem a mesma distribuição de $Y$. No contexto de simulação perfeita construímos dois ou mais processos estocásticos sobre um mesmo espaço de estados que evoluem obedecendo a mesma lei de transição.

Consideremos uma CM ergódica $\left\{X_{t}\right\}_{t \in \mathbb{Z}}$ estacionária com espaço de estados $\mathcal{X}$ (com $|\mathcal{X}|=N$, onde $|\mathcal{X}|$ denota a cardinalidade de $\mathcal{X}$ ) e matriz de transição $P$. A evolução de uma trajetória da CM $\left\{X_{t}\right\}_{t \in \mathbb{Z}}$ pode ser feita através de uma função de atualização $\phi$ e uma série de variáveis aleatórias $U_{t}$, onde $U_{t} \sim U(0,1)$, de tal modo que

$$
X_{t+1}=\phi\left(X_{t}, U_{t+1}\right)
$$

A seguir apresentamos um exemplo para compreender melhor do que é o acoplamento juntamente com o funcionamento de $\phi$ na Equação 2.1.

Exemplo 2.1.1 Considere a $C M\left\{X_{t}\right\}_{t \in \mathbb{Z}}$ com espaço de estados $\mathcal{X}=\{0,1,2\}$ e matriz de transição

$$
\mathbf{P}=\left[\begin{array}{lll}
0,583 & 0,333 & 0,083 \\
0,417 & 0,417 & 0,167 \\
0,278 & 0,444 & 0,278
\end{array}\right]
$$

Considere também a matriz de probabilidades acumuladas de $\left\{X_{t}\right\}_{t \in \mathbb{Z}}$

$$
\mathbf{C}=\left[\begin{array}{lll}
0,583 & 0,917 & 1,0 \\
0,417 & 0,833 & 1,0 \\
0,278 & 0,722 & 1,0
\end{array}\right]
$$

a função de transição $\phi$ é tal que:

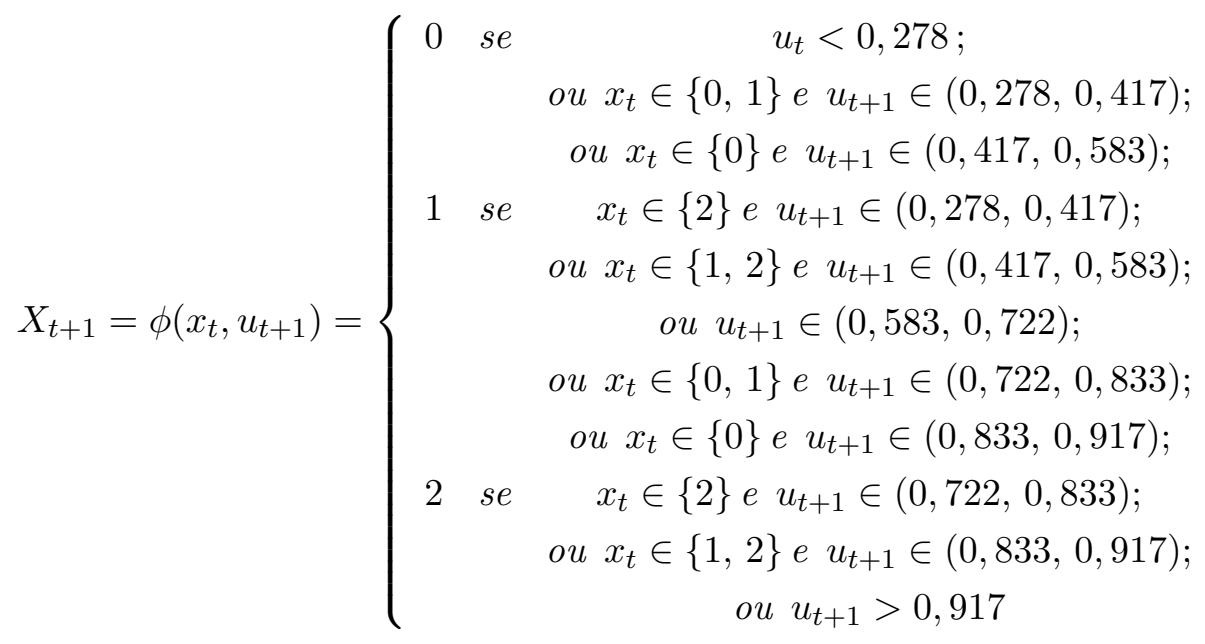

Matrizes de transição como apresentadas no Exemplo 2.1.1 são definidas apenas para casos em 
que o espaço de estados é discreto. Quando temos um espaço de estados contínuo trabalhamos com a função de probabilidade acumulada condicional de $X_{t}$ dado $X_{t-1}, F_{X_{t} \mid X_{T-1}=x_{t-1}}(\cdot)$. Lembramos que $F_{X_{t} \mid X_{T-1}=x_{t-1}}(\cdot)$ também pode ser definida para quando temos um espaço de estados discreto.

\subsubsection{Coalescência}

Discutimos a seguir o conceito mais importante para a construção do CFTP, a ideia de coalescência.

Dizemos que duas ou mais trajetórias de CM, partindo de estados quaisquer do espaço de estados $\mathcal{X}$, coalesceram se suas trajetórias se juntaram depois de um tempo aleatório $T$ e continuaram juntas após este tempo. Seja $X^{s, j} \equiv\left\{X_{t}^{s, j}\right\}_{t>s}$ a CM iniciada no tempo $s$ e no estado $j$, e assim o evento da coalescência de duas ou mais trajetórias de cadeias de Markov ocorre quando $X_{r}^{s, 1}=$ $X_{r}^{s, 2}=\ldots=X_{r}^{s, k} \Rightarrow X_{t}^{s, 1}=X_{t}^{s, 2}=\ldots=X_{t}^{s, k} \forall t>r$ para algum $t$.

Teorema 2.1.1 Suponha que temos $k$ cadeias de Markov, ergódicas, acopladas, onde $\forall j \in \mathcal{X}$ iniciamos uma cadeia $X^{s, j}$ e a atualização das mesmas é feita através de(2.1). Então;

1. O tempo T para ocorrer a coalescência é uma variável aleatória que depende somente da sequência de variáveis aleatórias uniformes $U_{1}, U_{2}, \ldots$

2. A variável aleatória $X_{0}$ (o valor comum na coalescência) é independente do valor inicial da cadeia.

A prova do Teorema 2.1.1 encontra-se em Casella, Lavine \& Robert (2001).

Em síntese, o CFTP é um algoritmo cujos resultados são o tempo $-T$, tal que a coalescência ocorra entre o tempo $-T$ e o tempo 0 , e um estado $x_{0}$, valor comum das trajetórias coalescidas no tempo 0 , que é um valor exato da distribuição limite $\pi$.

\subsection{Implementação do CFTP}

Nesta seção apresentamos um exemplo de Casella et al. (2001) que ilustra o funcionamento do CFTP, de uma forma mais didática, para o caso em que $\mathcal{X}$ é discreto e finito.

Exemplo 2.2.1 Considere o modelo Beta-Binomial, com hiper-parâmetros n, $\alpha, e \beta$, fixados, dado por $\Theta \sim \operatorname{Beta}(\alpha, \beta)$ e $X \mid \Theta=\theta \sim \operatorname{Binomial}(n, \theta)$ gerando a distribuição conjunta $\pi(x, \theta)=\frac{\left(\begin{array}{c}n \\ x\end{array}\right)}{B(\alpha, \beta)} \theta^{x+\alpha-1}(1-\theta)^{n-x+\beta-1}$, onde $B(m, n)=\int_{0}^{1} t^{m-1}(1-t)^{n-1}$ denotando a função beta. É fácil notar que a densidade condicional de $\theta \mid X=x$ é uma Beta $(\alpha+x, \beta+n-x)$, e que a função de probabilidade marginal de $X$ da forma $P(X=x)=\left(\begin{array}{l}n \\ x\end{array}\right) \frac{B(\alpha+x, \beta+n-x)}{B(\alpha, \beta)}$ caracterizando a distribuição beta binomial com parâmetros $n, \alpha$ e $\beta$

Podemos construir uma CM tendo $\pi$ como densidade estacionária usando a seguinte regra de transição para $\left(X_{t}, \Theta_{t}\right) \rightarrow\left(X_{t+1}, \Theta_{t+1}\right)$

1. $\operatorname{Gerar} \Theta_{t+1} \mid X_{t}=x_{t} \sim \operatorname{Beta}\left(\alpha+x_{t}, \beta+n-x_{t}\right)$, $e$

2. $\operatorname{Gerar} X_{t+1} \mid \Theta_{t+1}=\theta_{t+1} \sim \operatorname{Binomial}\left(n, \theta_{t+1}\right)$ 
Considerando somente a sub-cadeia $\ldots, X_{t}, X_{t+1}, \ldots$ e calculando a função de transição temos que $\left\{X_{t}\right\}_{t \in \mathbb{Z}}$ é uma CM com $X_{t+1} \mid X_{t}=x_{t} \sim \operatorname{BetaBinomial}\left(n, \alpha+x_{t}, \beta+n-x_{t}\right)$ e um núcleo de transição $K\left(x_{t}, x_{t+1}\right) \propto\left(\begin{array}{c}n \\ x_{t=1}\end{array}\right) \frac{\Gamma(\alpha+\beta+n) \Gamma\left(\alpha+x_{t}+x_{t+1}\right) \Gamma\left(\beta+2 n-x_{t}-x_{t+1}\right)}{\Gamma\left(\alpha+x_{t}\right) \Gamma\left(\beta+n-x_{t}\right) \Gamma(\alpha+\beta+2 n)}$. A matriz de transição $\mathbf{P} d o$ exemplo 2.1.1 foi montada com essa regra de transição com $n=2, \alpha=2, e \beta=4$

Usando os hiper-parâmetros que geraram as matrizes $\boldsymbol{C}$ e $\mathbf{P}$ do exemplo 2.1.1 seguiremos o seguinte algoritmo para gerar uma amostra exata da distribuição estacionária de $X_{t}$ :

1. Começar as cadeias $X^{-1,1}, X^{-1,2}, \ldots, X^{-1, k}$ no tempo $t=-1$ para cada estado de $\mathcal{X}$ e gerar $U_{0}$

2. Atualizar cada cadeia para o tempo $t=0$ aplicando a $X_{0}^{-1, j}=\phi\left(X_{-1}^{-1, j}, U_{0}\right)$. Se ocorrer a coalescência no tempo $t=0$, então $T=-1$ e $x_{0}$, o valor comum das cadeias coalescidas no tempo zero, é um valor de $\pi$.

3. Caso contrario, voltar até o tempo $t=-2$ começar as cadeias $X^{-2,1}, X^{-2,2}, \ldots, X^{-2, k}$, e atualizar cada cadeia usando $X_{-1}^{-2, j}=\phi\left(X_{-2}^{-2, j}, U_{1}\right)$ e $X_{0}^{-2, j}=\phi\left(X_{-1}^{-2, j}, U_{0}\right)$. Se ocorrer a coalescência no tempo $t=0$, então $T=-2$ e $x_{0}$ é um valor de $\pi$.

4. Se não houver coalescência, repete-se o processo até encontrar um tempo $T$ tal que ocorra coalescência entre o tempo - $T$ e o tempo 0

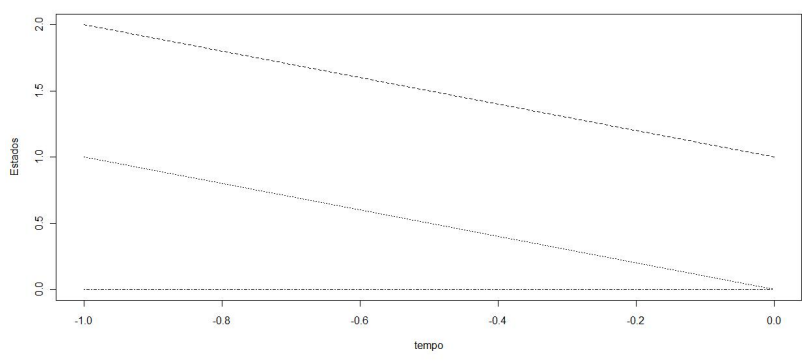

Figura 2.1: Trajetórias de cadeias de Markov atualizadas para $T=-1$

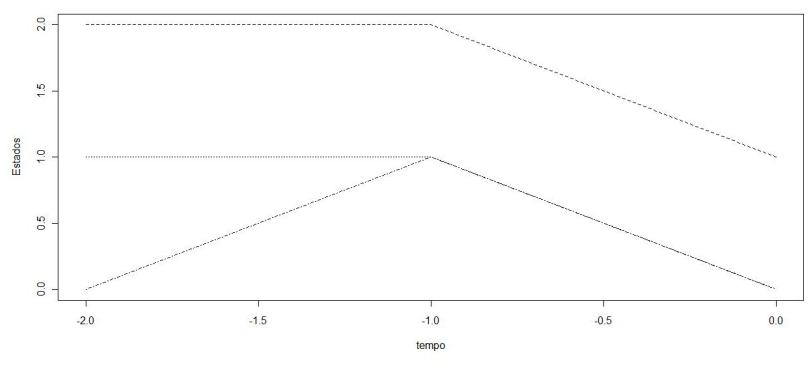

Figura 2.2: Trajetórias de cadeias de Markov atualizadas para $T=-2$ 


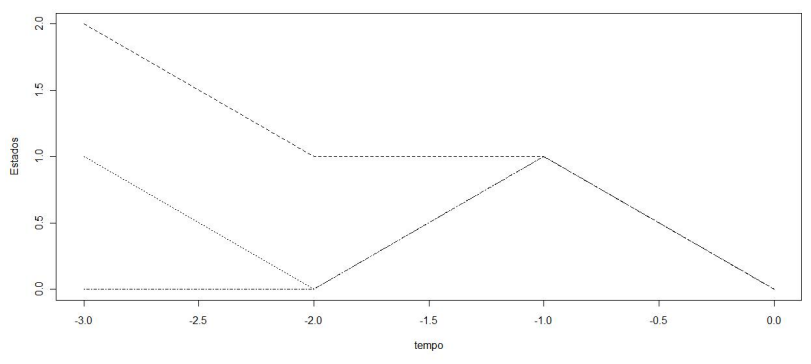

Figura 2.3: Trajetórias de cadeias de Markov atualizadas para $T=-3$

Ilustramos uma realização do CFTP para o modelo beta binomial descrito acima, utilizando $n=$ $2, \alpha=2, \beta=4$. Neste primeiro passo foi gerado um número aleatório $U_{0}=0,2875$, e usando a função de atualização 2.3 descrita no Exemplo 2.1.1, temos o resultado apresentado na Figura 2.1. A trajetória iniciada no estado 0 coalesceu com a trajetória iniciada no estado 1, mas não houve coalescência de todas as trajetórias. Fazemos $T=-2$, geramos um novo número aleatório, $U_{1}=$ 0,7883 , atualizamos as trajetórias para $t=-1$ aplicando $U_{1}$ em 2.3 e em seguida para $t=0$ aplicando $U_{0}$ em 2.3, o resultado desse estagio está representando na Figura 2.2. Novamente as trajetórias inciadas nos estados 0 e 1 coalesceram no tempo $t=-1$, mas o algoritmo não chegou ao fim, pois as trajetórias não coalesceram por completo. Fazemos $T=-3$, geramos um número aleatório, $U_{2}=0,4089$, e atualizamos para $t=-2$ e $t=-1$. Na Figura 2.3 vemos que as trajetórias iniciadas em 0 e 1 coalesceram em um passo, e a trajetória resultante coalesce com a trajetória iniciada em 2 no passo seguinte e para finalizar o algoritmo evoluímos a trajetória resultante a para $t=0$ e obtivemos um valor $X_{0}=0$ que é uma amostra de $\pi$. Note que, a sequencia de números aleatórios, $U_{0} U_{1}$ e $U_{2}$, permanece a mesma durante toda a execução do algoritmo.

\subsection{CFTP monótono}

Nesta seção apresentaremos uma construção do algoritmo CFTP para configurações especificas de $\mathcal{X}$.

Considere uma CM, ergódica, com espaço de estados $\mathcal{X}=\{0,1,2, \ldots, 99,100\}$. De acordo com a definição do algoritmo CFTP temos que iniciar uma trajetória para cada um dos estados pertencentes a $\mathcal{X}$,isto é, criar 101 trajetórias de cadeias de Markov, atualizá-las de acordo com a série de variáveis aleatórias uniformes, de um tempo $t$ até o tempo 0, até encontrar um certo tempo $T$ tal que a coalescência ocorra entre o tempo $T$ e o tempo 0 . Tal processo é dispendioso, computacionalmente falando. Para tal caso, existe uma versão do CFTP, o CFTP-monótono, que consiste em escolher somente duas possíveis trajetórias com propriedades especiais e verificar a coalescência somente destas duas trajetórias. A seguir apresentamos algumas definições para auxiliar na construção do algoritmo CFTP-monótono.

Definição 2.3.1 (Relação de ordem parcial estrita "ミ”) Considere uma relação $\preceq$. Uma relação é relação de ordem parcial estrita quando satisfaz as seguintes condições: 
- $a \preceq b$ e $b \preceq a$ não podem acontecer simultaneamente, $e$

- $\preceq$ é transitiva

Definição 2.3.2 (Conjunto parcialmente ordenado) Um conjunto $V$ é chamado de parcialmente ordenado se uma relação de ordem parcial estrita " $\preceq$ " é definida nele. As propriedades fundamentais de um conjunto parcialmente ordenado são:

- se $a \preceq b e b \preceq c$ então $a \preceq c \forall a, b, c \in V$

- $a \preceq b$ e $b \preceq a$ não pode acontecer simultaneamente

As Definições 2.3.1 e 2.3.2 auxiliam no entendimento da definição do CFTP-monótono, pois como será mostrado mais adiante, necessitamos de um espaço de estados cujos elementos possam ser ordenados de acordo com alguma medida de probabilidade, seja esta, uma matriz ou uma função de transição. Segue a definição do CFTP-monótono como vista em Coelho (2001)

Definição 2.3.3 a) Seja $P$ uma matriz de transição de uma CM em um espaço de estados parcialmente ordenado $(\mathcal{X}, \preceq)$. Dizemos que a cadeia é monótona se $P$ preservar a ordem parcial, isto é, se

$$
P(x, \cdot) \leq P(y, \cdot)
$$

estocasticamente se $x \preceq y$

b) Dizemos que uma função de atualização $\phi$ associada a $P$ é monótona se para $x \preceq y$, temos que $\phi(x, u) \preceq \phi(y, u)$ quase certamente para $u \in[0,1]$.

A Definição 2.3.3 se refere a um espaço de estados discreto e finito, ou infinito enumerável, pois trata de matriz de transição, que é definida somente para $\mathcal{X}$ discreto, mas a definição pode ser estendida para casos em que $\mathcal{X}$ é contínuo, como mostraremos mais adiante. Para isso basta trocar a matriz de transição por uma função de transição semelhante a equação 2.1, com certas características presente nas funções de transições apresentadas em Murdoch \& Green (1998). Note que $\phi$ é definido para ambos os casos $\mathcal{X}$ discreto ou contínuo. No exemplo 2.1.1 é apresentada uma função de transição para o caso $\mathcal{X}$ discreto.

Suponha também que $\mathcal{X}$ tenha estados máximo $(\hat{1})$ e mínimo $(\hat{0})$ tal que $\hat{0} \preceq x \preceq \hat{1} \forall x \in \mathcal{X}$. Então, devido a monotonicidade, o CFTP necessita somente da verificação da coalescência entre as trajetórias partindo dos estados $\hat{1}$ e $\hat{0}$ para verificarmos a coalescência completa, pois as trajetórias de $\hat{0}$ e $\hat{1}$ englobam as outras trajetórias.

\subsection{CFTP para $\mathcal{X}$ contínuo}

Propp \& Wilson criaram o CFTP e provaram o fato do mesmo produzir uma amostra que segue exatamente a distribuição de interesse, definida como $\pi$. A seguir, enunciamos os três teoremas, contidos no trabalho Propp \& Wilson (1996), que combinados dão suporte teórico para o funcionamento do CFTP no caso em que a distribuição tem suporte discreto. Murdoch \& Green 
(1998) apresenta argumentos e certas características da função de transição $\phi$ para que o terceiro teorema tenha validade para quando $\mathcal{X}$ é contínuo. Propp \& Wilson (1996) utilizam argumentos interessantes, sem efetuar cálculos matemáticos, e por isso apresentaremos a seguir os três teoremas, seguidos de suas respectivas provas, e a transcrição dos argumentos de Murdoch \& Green (1998) para a validade do CFTP quando $\mathcal{X}$ é contínuo.

Para facilitar o entendimento nesta seção usaremos a notação empregada em Propp \& Wilson (1996) e Murdoch \& Green (1998). $f_{t}(i)$ (o mesmo que $X_{t+1}$ ) é o valor assumido por uma determinada CM no instante $t+1$, sendo que no instante $t$ a $\mathrm{CM}$ em questão estava no estado $i$. A composição $f_{t_{2}-1} \circ f_{t_{2}-2} \circ \ldots \circ f_{t_{1}+1} \circ f_{t_{1}}$ representada a trajetória de uma CM entre o instante $t_{1} \mathrm{e}$ o instante $t_{2}$, denotada por $F_{t_{1}}^{t_{2}}$, onde $t_{2}>T_{1}$. Uma trajetória se torna constante se $F_{t}^{0}(i)=F_{t}^{0}\left(i^{\prime}\right)$ para todo $i, i^{\prime}$

Teorema 2.4.1 Com probabilidade 1 o algoritmo CFTP retorna algum número, e esse número é o possivel valor de uma variável aleatória que é distribuído de acordo com a distribuição estacionária da $C M$.

Idéia da prova A CM em questão sendo ergódica, existe um $L$ tal que para todos estados $i$ e $j$, existe uma probabilidade positiva de ir do estado $i$ para o estado $j$ em $L$ passos. Então para cada $t, F_{t-\mathbf{L}}^{t}(\cdot)$ tem uma probabilidade positiva de ser constante. Desde de que cada uma das trajetórias $F_{-L}^{0}(\cdot), F_{-2 L}^{-L}(\cdot), \ldots$ tem uma probabilidade positiva $\epsilon>0$ de ser constante, e como estes eventos são independentes, então com probabilidade 1 um destes mapas é constante, em tal caso $F_{-M}^{0}$ é constante para todo $M$ suficientemente grande. Quando o algoritmo voltar $M$ passos no passado, irá terminar e retornará um valor, chamaremos de $\overline{F_{-\infty}^{0}}$. Note que $\overline{F_{-\infty}^{0}}$ é obtido através de $\overline{F_{-\infty}^{-1}}$ evoluindo a CM um passo, e que $\overline{F_{-\infty}^{0}}$ e $\overline{F_{-\infty}^{0}}$ tem a mesma distribuição de probabilidade. Juntamente essas duas afirmações implicam que o resultado $\overline{F_{-\infty}^{-1}}$ é distribuído de acordo com a única distribuição estacionária.

Teorema 2.4.2 Sejam $\ldots, U_{-3}, U_{-2}, U_{-1}$ variáveis aleatórias i.i.d., e $\phi(\cdot, \cdot)$ uma função determinística com a propriedade de que para todo $\boldsymbol{i}, \operatorname{Prob}\left[\phi\left(i, U_{-1}\right)=j\right]=p_{i, j}$. Definimos $f_{t}(i)=$ $\phi\left(i, U_{t}\right)$ e $F_{t}^{0}=f_{-1} \circ f_{-2} \circ \ldots \circ f_{t}$. Assumimos que com probabilidade 1 , existe um $\boldsymbol{t}$ para qual $a$ trajetória $F_{t}^{0}$ é constante, com um valor constante que denotaremos por $\phi\left(\ldots, U_{-2}, U_{-1}\right)$. Então a variável aleatória $\phi\left(\ldots, U_{-2}, U_{-1}\right)$, que é definida com probabilidade 1 , segue a distribuição $\pi$.

Ao invés de dar a prova do resultado acima, Propp \& Wilson (1996) enunciam e provam um um outro resultado que generaliza o Teorema 2.4.2, além de ser mais próximo do procedimento utilizado na prática

Teorema 2.4.3 Seja $\ldots, U_{-3}, U_{-2}, U_{-1}$ variáveis aleatórias e seja $\phi_{t}(\cdot, \cdot)(t<0)$ uma sequencia de funçôes determinísticas com a propriedade para todo $i$ e $j$,

$$
\sum_{i} \pi(i) \operatorname{Prob}\left[\phi_{t}\left(i, U_{t}\right)=j\right]=\pi(j)
$$


Definimos $f_{t}(i)=\phi_{t}\left(i, U_{t}\right)$ e $F_{t}^{0}=f_{-1} \circ f_{-2} \circ \ldots \circ f_{t}$. Assumimos que com probabilidade 1 , existe um t para qual a trajetória $F_{t}^{0}$ é constante, com um valor constate denotado por $\phi\left(\ldots, U_{-2}, U_{-1}\right)$. Então a variável aleatória $\phi\left(\ldots, U_{-2}, U_{-1}\right)$, que é definida com probabilidade 1 , segue a distribuição $\pi$

Idéia da prova Seja $X$ a variável aleatória definida no espaço de estados de uma CM governada pela distribuição estacionária $\pi$, e para todo $t<0$ seja $Y_{t}$ a variável aleatória $F_{t}^{0}(X)$, a trajetória da variável $X$. Cada $Y_{t}$ tem distribuição $\pi$, e a sequência $Y_{-1}, Y_{-2}, Y_{-3}, \ldots$ converge quase certamente para algum estado $Y_{-\infty}$ que também deve ter distribuição $\pi$. Use $\phi\left(\ldots, U_{-2}, U_{-1}\right)=Y_{-\infty}$

Murdoch \& Green (1998) assumem que o espaço de estados é $\mathcal{X} \subset \mathbb{R}^{d}$ e que o núcleo de transição de interesse e a distribuição estacionária têm densidades com respeito a medida de Lebesgue $d$ dimensional. Eles escrevem a densidade de $X_{t+1}$ no ponto $y$, dado $X_{t}=x$ como $f(y \mid x)$ e a distribuição estacionária como $\pi(\cdot)$. Escrevem também a versão contínua da equação 2.4.

Neste mesmo trabalho Murdoch \& Green relaxam a afirmação da existência de $f$, a função de densidade de probabilidade, implicando que a distribuição de interesse não necessite ser absolutamente contínua, de modo a considerar amostradores de Metropolis-Hastings, e também apresentam um algoritmo ,transcrito mais abaixo, que serve de base para todos os outros algoritmos contidos em Murdoch \& Green (1998), como por exemplo o algoritmo do acoplador Multigamma, além de ilustrar as características necessárias para que uma função determinística $\phi$ possa validar o Teorema 2.4.3 quando $\mathcal{X}$ e contínuo.

Uma vez definida uma função determinística $\phi$ que satisfaça a Equação 2.1 e que siga o algoritmo apresentado mais adiante, a prova de que o algoritmo, quando existe, retornar um valor, e esse valor seja uma amostra exata da distribuição desejada é similar a prova do teorema 2.4.3 reescrita para um espaço de estados contínuo.

Com os argumentos citados aqui, Murdoch \& Green (1998), em síntese afirmam que uma função de atualização capaz de evoluir uma CM com espaço de estados contínuos dependendo somente do estado anterior e de uma variável aleatória $U_{t}$, torna o CFTP eficiente e com validade para gerar amostras exatas de distribuições contínuas. 


$$
\int \pi(x) f(y \mid x) d x=\pi(y)
$$

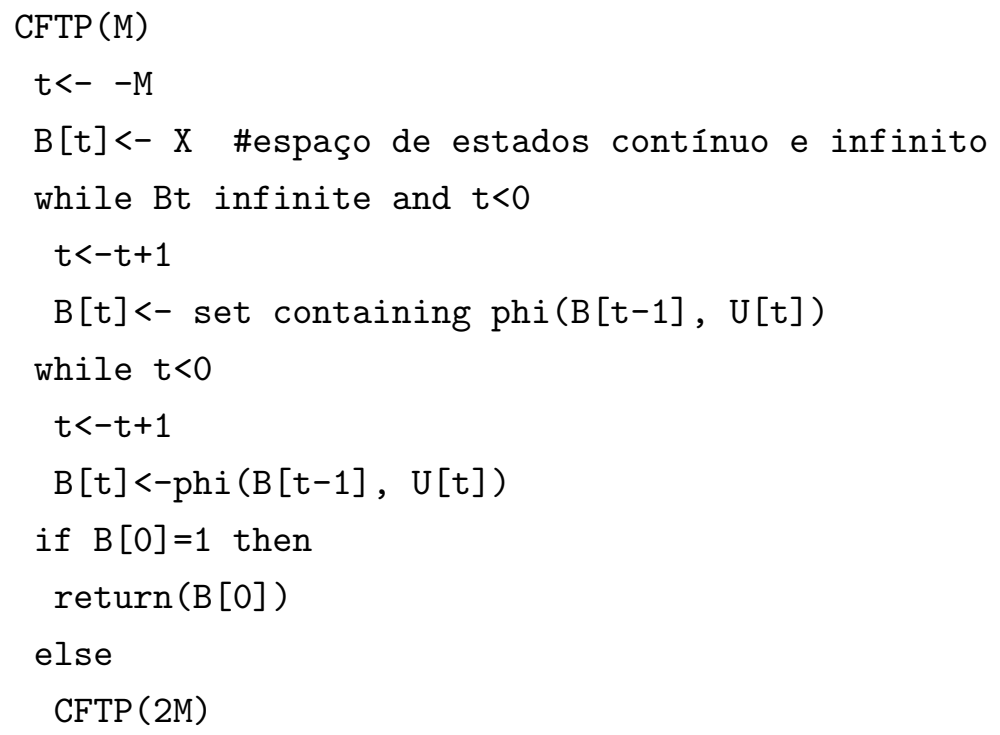

Exemplo 2.4.1 (Murdoch ÉG Green 1998) Suponha que a densidade do núcleo de transição, $f(\cdot \mid x)$, de uma $C M$ é conhecida. $f(\cdot \mid x)$ representa a densidade condicional de $X_{t+1}$ dado $X_{t}=x$. Suponha também que

$$
f(y \mid x) \geq r(y)
$$

para alguma função não negativa $r(\cdot)$ para a qual $\rho=\int_{\mathcal{X}} r(y) d y$, por questão de notação considere y unidimensional. Considere

$$
\begin{aligned}
R(y) & =\rho^{-1} \int_{-\infty}^{y} r(v) d v \\
Q(y \mid x) & =(1-\rho)^{-1} \int_{-\infty}^{y}[f(v \mid x)-r(v)] d v .
\end{aligned}
$$

O acoplador multi-gama é aquele que tem uma função de transição $\phi$ da forma:

$$
\phi(x, U)=\left\{\begin{aligned}
R^{-1}\left(U^{(1)}\right) & \text { se } U^{(1)}<\rho \\
Q^{-1}\left(U^{(2)} \mid x\right) & \text { caso contrario }
\end{aligned}\right.
$$

onde o par $U=\left(U^{(1)}, U^{(2)}\right)$ é um vetor de variáveis aleatórias.

Tendo em vista que o algoritmo CFTP contínuo funciona então a versão monótona do CFTP contínuo funciona, desde que a densidade do núcleo de transição seja monótona. 


\section{Capítulo 3}

\section{Simulação da normal bivariada truncada}

Neste capítulo apresentaremos três métodos para a simulação de valores de acordo com a distribuição normal bivariada truncada. Estes métodos são: o algoritmo de rejeição, o amostrador de Gibbs(Gibbs Sampler) e o amostrador perfeito(Perfect Sampler) mais especificamente o algoritmo CFTP.

A distribuição normal bivariada truncada tem densidade de probabilidade:

$$
f_{X, Y}(x, y)=\frac{e^{-\frac{1}{2}[(x y)-\mu]^{t} \Sigma^{-1}[(x y)-\mu]} I_{\{A\}}(x, y)}{\int_{A} e^{-\frac{1}{2}[(x y)-\mu]^{t} \Sigma^{-1}[(x y)-\mu]} I_{\{A\}}(x, y) d x d y}
$$

onde $\mu$ é o vetor de médias e $\Sigma$ é a matriz de variância e covariância, ambos referentes a distribuição original, antes do trucamento.

O algoritmo de rejeição consiste em simular valores de uma densidade de probabilidade $f$ através de uma outra densidade $g$ tal que $g(i) \geq f(i)$ para todo $i \in \mathbb{R}$. Para simular valores da distribuição normal bivariada truncada, $f$, utilizaremos a distribuição normal bivariada anterior ao truncamento, g. Primeiro calculamos a taxa de aceitação $(\alpha)$ que depende da região de valores possíveis da distribuição truncada, denotada por $A$. Quanto maior for $A$ maior vai ser a taxa de aceitação, quanto menor for $A$ menor a taxa de aceitação. Uma taxa de aceitação pequena implica em uma demora para se gerar amostras e também na perda de informação, pois serão necessárias mais valores rejeitados para que tenhamos um valor aceito como amostra da distribuição de interesse.

O amostrador de Gibbs é um algoritmo MCMC que gera variáveis aleatórias de uma densidade de probabilidade indiretamente, sem conhecer completamente a função densidade. Sua essência constituí em simular uma CM, cuja distribuição estacionária é a distribuição de interesse. A CM é inicializada em um ponto qualquer pertencente ao conjunto de valores possíveis da mesma e, após um período de aquecimento, onde gera-se observações "suficientes" para considerar a convergência da mesma a amostra é construída. Usualmente respeita-se um certo espaçamento entre os valores simulados para tentar evitar a dependência entre os valores. A amostra gerada por algoritmos MCMC são consideradas como uma aproximação, expectativa do que espera-se obter de uma real amostra da distribuição de interesse. Na maioria dos casos a aproximação da amostra é considerada boa, podendo, a amostra em questão, ser utilizada como objeto de estudo. Mais claramente, o algoritmo MCMC, em geral, consiste em: 
1. Determinar, arbitrariamente, um período de aquecimento(burning time) e um espaçamento entre as observações.

2. Escolher um valor inicial, $x_{0}$.

3. Evoluir a cadeia de Markov partindo de $x_{0}$, simulando valores da cadeia até simularmos uma quantidade de valores igual ao número estipulado pelo período de aquecimento.

4. Continuar evoluindo a cadeia e guardar os valores simulados após o período de aquecimento, respeitando o espaçamento entre os mesmo.

5. Quando tivermos uma quantidade de valores simulados suficiente para nossa amostra paramos de evoluir a cadeia.

O amostrador de Gibbs se diferencia do algoritmo MCMC geral pelo fato de usar as condicionais completas, distribuições condicionais de uma das componentes da variável multivariada em estudo com relação com as demais. Por exemplo, se $\mathbf{X}=\left(X_{1}, X_{2}, X_{3}\right)$ as distribuições condicionais $X_{1}\left|X_{2}=x_{2}, X_{3}=x_{3}, X_{2}\right| X_{1}=x_{1}, X_{3}=x_{3}$ e $X_{3} \mid X_{1}=x_{1}, X_{2}=x_{2}$ são as condicionais completas. Em cada interação do amostrador de Gibbs, quando evoluímos as cadeias, evoluirmos uma condicional completa por vez. Na iteração $i+1$ sorteamos um valor $x_{(i+1)} 1$ da distribuição $X_{(i+1) 1} \mid X_{i 2}=x_{i 2}, X_{i 3}=x_{i 3}$, um valor $x_{(i+1) 2}$ da distribuição $X_{(i+1) 2} \mid X_{(i+1) 1}=x_{(i+1) 1}, X_{i 3}=$ $x_{i 3}$ e um valor $x_{(i+1) 3}$ da distribuição $X_{(i+1) 3} \mid X_{(i+1) 1}=x_{(i+1) 1}, X_{(i+1) 2}=x_{(i+1) 3}$. O vetor $\left(x_{(i+1) 1}, x_{(i+1) 2}, x_{(i+1) 3}\right)$ é o valor da CM na iteração $i+1$.

Casella \& George (1992) apresentam mais detalhadamente a mecânica do amostrador de Gibbs.

Este algoritmo apresenta problemas para se determinar o momento da convergência da cadeia de Markov, pois de acordo com a teoria de cadeias de Markov, uma cadeia converge após infinitas transições, e nem sempre se consegue determinar um período de aquecimento suficientemente grande.

Em Robert (1995) é apresentado um algoritmo para simular valores da distribuição normal multivariada truncada usando o amostrador de Gibbs. Neste artigo percebe-se que a distribuição condicional completa para cada componente, $X_{i}$ do vetor $\bar{X}$, se refere a um conjunto de truncamento $A_{i}$ especifico. Para simular um ponto possível do vetor $\bar{X}$ acaba-se por simular p valores unidimensionais, onde cada valor $X_{i}$ tem seu próprio conjunto de valores possíveis. O pacote tmvtnomr do programa estatístico $\mathbf{R}$ traz rotinas otimizadas para a simulação de valores da distribuição normal multivariada truncada utilizando estes dois algoritmos, rejeição e Gibbs.

\subsection{Amostrador perfeito}

No Capítulo 2 foi descrito um algoritmo de simulação perfeita, o algoritmo CFTP, que utiliza o acoplamento de cadeias de Markov para gerar valores que realmente obedecem a lei da distribuição de interesse, diferentemente da amostra MCMC, que é uma amostra aproximada da distribuição de interesse.

Devido ao fato citado acima, o amostrador perfeito leva vantagem sobre o amostrador de Gibbs, pois não precisa de um período de aquecimento e nem de um espaçamento entre as observações para 
obter uma amostra com valores independentes entre si. $\mathrm{O}$ amostrador perfeito, mais especificamente o CFTP, gera um único valor para cada vez que é executado. Para gerar uma amostra com mais de um elemento, executa-se o CFTP um número suficiente de vezes. Como o acoplamento de cadeias de Markov permite que o valor gerado pelo CFTP seja independente do valor inicial das cadeias, tem-se a independência dos valores amostrais gerados pelas diversas execuções do CFTP.

Philippe \& Robert (2003) apresentam o CFTP configurado para simular amostras da distribuição normal bivariada truncada no quadrante positivo. Este algoritmo necessita somente que seja informado o vetor de médias $(\mu)$, a matriz de variância e covariância $(\Sigma)$, ambos oriundos da distribuição normal anterior ao truncamento, e um vetor informando o valor que minimiza a densidade no conjunto de truncamento( $(\hat{0})$, quadrante positivo no caso.

O valor que maximiza a distribuição no conjunto de truncamento(1̂) é calculada dentro do próprio algoritmo. Se $\mu \in \mathcal{X}$ então $\hat{1}=\mu$, caso contrario

$$
\hat{1}=\operatorname{argmax}_{x \in \mathcal{M}^{+}}(x-\mu)^{t} \Sigma^{-1}(x-u)
$$

onde

$$
\mathcal{M}^{+}=\left\{(0,0),\left(\mu_{1}+\mu_{2} \frac{\sigma_{12}}{\sigma_{22}}, 0\right),\left(0, \mu_{2}+\mu_{1} \frac{\sigma_{12}}{\sigma_{11}}\right)\right\} \bigcap\left(\mathbb{R}_{+}\right)^{2}
$$

\subsubsection{Evolução das cadeias de Markov e função de transição}

Para facilitar a leitura, considere que todas as variáveis descritas aqui são bivariadas

No algoritmo CFTP, para a evolução das cadeias de Markov é utilizado uma função de atualização $\phi$ baseada no algoritmo de aceitação e rejeição, ver Murdoch \& Green (1998). Tal função tem a seguinte forma:

$$
X_{t+1}=\phi\left(U_{t}, x_{t}\right)=z \text {, onde } z \in\left\{z ; f_{X}(z) \geq U_{t} f_{X}\left(x_{t}\right)\right\}
$$

Em outras palavras, sorteia-se, aleatoriamente, elementos da distribuição até que seja sorteado um elemento que satisfaça a condição $f_{X}(z) \geq U_{t} f_{X}\left(x_{t}\right)$, onde $f_{X}$ é a lei da distribuição. Como $f_{X}$ tem a forma da distribuição normal, a desigualdade na equação 3.2 é resumida a

$$
\left[X_{t+1}-\mu\right]^{t} \Sigma^{-1}\left[X_{t+1}-\mu\right]<K_{t+1}
$$

onde $K_{t+1}=-2 \log \left(U_{t+1}\right)+\left[x_{t}-\mu\right]^{t} \Sigma^{-1}\left[x_{t}-\mu\right]$, ou ainda

$$
Y_{t+1}^{t} \Lambda^{-1} Y_{t+1}<K_{t+1}
$$

onde $Y=Q^{t}(X-\mu)$ e $\Sigma^{-1}=Q \Lambda^{-1} Q^{t}$.

Pelo fato da variável $Y$ possuir componentes independentes, pois a matriz $\Lambda$ é uma matriz diagonal, e vetor de medias $(0,0)^{t}$, é mais fácil trabalhar com essa variável, ao invés da variável original. Perceba que os valores que satisfazem a inequação (3.2) estão contidos em uma elipse centrada na origem, isso ocorre devido ao uso da distribuição normal e a transformação de variável. 
Com a elipse formada, de acordo com o algoritmo de aceitação e rejeição, construímos uma caixa que engloba este elipsoide, cujas arestas tangenciam a elipse, e simulamos pontos uniformes dentro dessa caixa. Caso o valor simulado esteja contido na elipse, verificamos se o mesmo pertence ao $\mathbb{R}_{+}^{2}$, caso contrario sorteia-se um novo valor dentro da caixa. O processo continua até ser sorteado um valor que esteja contido na elipse e pertença ao $\mathbb{R}_{+}^{2}$.

Na figura 3.1 está representada a caixa e o elipsoide quando temos, antes do truncamento $\mu=\left(\begin{array}{l}2 \\ 2\end{array}\right), \Sigma=\left[\begin{array}{cc}2.5 & -2.5 \\ -2.5 & 3.5\end{array}\right], u_{t}=0.2296152$ e $x_{t}=\left(\begin{array}{c}10 \\ 10\end{array}\right)$ e $U_{t+1}=0.2296152$

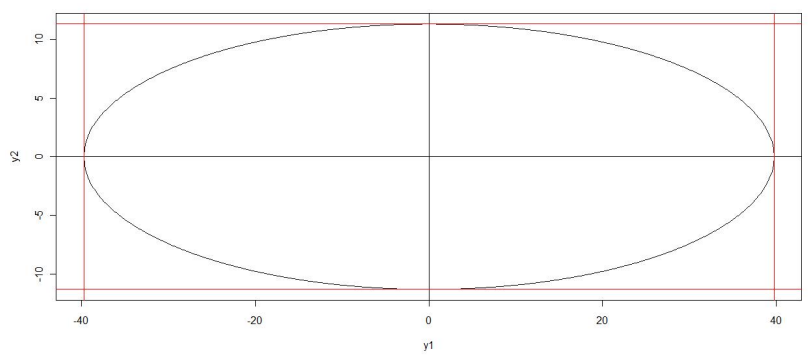

Figura 3.1: Caixa e elipsoide gerados pelo algoritmo de aceitação e rejeição

Os demais detalhes do CFTP descrito em Philippe \& Robert (2003) são semelhantes ao CFTP original.

Ao implementarmos o CFTP notamos certas particularidades sobre a caixa na qual executamos o algoritmo de aceitação e rejeição. Philippe \& Robert (2003) mostram manipulações algébricas para reduzir a caixa, deixando-a menor e totalmente dentro da elipsoide. Tal manipulação pode diminuir o tempo gasto pelo algoritmo. Mas, dependendo da localização do vetor de médias posterior ao truncamento, essa redução na caixa pode aumentar o tempo computacional, como, por exemplo, quando o vetor de médias esta no terceiro quadrante. Outra observação sobre a redução da caixa é que uma manipulação errônea dessa redução pode causar um viés nas amostras. 


\section{Capítulo 4}

\section{Análise dos dados simulados}

No presente capítulo faremos uma análise gráfica dos dados simulados através dos algoritmos CFTP, amostrador de Gibbs e método da rejeição, bem como uma comparação entre o tempo utilizado, por cada método, para gerar as amostras.

Todas as simulações foram feitas utilizando o programa $\mathbf{R}$ versão 2.10 .0 no sistema operacional Linux Ubuntu 8.04.3 LTS Kernel 2.6.24-24-rt em modo texto, sem um ambiente gráfico carregado de modo a ter todo o processamento da CPU voltado para as simulações. Os algoritmos foram executados em um computador Intel(R) Core $^{T M} 2$ Quad Q6600 de 2.4Ghz com 4gb de memoria RAM.

Para a simulação dos dados via CFTP foi implementado o algoritmo apresentado no Capítulo 3. Para a simulação dos dados via amostrador de Gibbs e método de rejeição, foi utilizada a função rtmvnorm do pacote tmvtnorm. Esta função(rtmvnorm) gera valores da distribuição normal multivariada truncada, nos permitindo escolher qual algoritmo usar, Gibbs ou rejeição, através do argumento algorithm.

Foram geradas amostras de quatro distribuições normais truncadas no quadrante positivo, com a mesma matriz de variância e covariância $\Sigma=\left[\begin{array}{ll}3.5 & 3.5 \\ 3.5 & 4.5\end{array}\right]$, mas com médias diferentes, $\mu_{1}=\left(\begin{array}{c}0.5 \\ 0.5\end{array}\right), \mu_{2}=\left(\begin{array}{c}-0.5 \\ 0.5\end{array}\right), \mu_{3}=\left(\begin{array}{c}-0.5 \\ -0.5\end{array}\right)$ e $\mu_{4}=\left(\begin{array}{c}0.5 \\ -0.5\end{array}\right)$. Note que gerar pontos amostrais somente no primeiro quadrante de uma distribuição que possui o vetor de médias fora do primeiro quadrante é equivalente ao problema de gerar valores na cauda de uma distribuição de probabilidade. Como o objetivo é verificar o desempenho de cada algoritmo para gerar pontos nas situações extremas, regiões de caudas de distribuição, por esse motivo temos essas quatro casos diferentes:

Caso 1: média no primeiro quadrante.

Caso 2: média no segundo quadrante.

Caso 3: média no terceiro quadrante.

Caso 4: média no quarto quadrante. 


\subsection{Análise gráfica}

Para cada distribuição foram geradas, através de cada um dos 3 algoritmos, dez amostras(réplicas) de tamanho mil. As réplicas tiveram comportamento semelhante, para cada distribuição amostrada e para cada algoritmo implementado, exceto uma réplica de amostra da terceira distribuição, vetor de médias anterior ao truncamento $\mu_{3}$ e matriz de variância e covariância anterior ao truncamento $\Sigma$, por esse motivo apresentaremos graficamente comparações de uma das réplicas de cada caso e de cada algoritmo, e também o gráfico referente a réplica citada acima. Em todos os valores gerados pelo CFTP o valor de 0 foi definido como sendo o vetor $\left(\begin{array}{l}10 \\ 10\end{array}\right)$, ou seja, consideramos esse ponto como o ponto que minimiza a distribuição de interesse, truncando assim o quadrante positivo, pois a probabilidade acumulada, apos o ponto $\hat{0}$, é muito pequena. Para os valores gerados pelo amostrador de Gibbs foram definidos mesmo período de aquecimento, dez mil, e um espaçamento de 100 valores gerados entre cada observação.

No primeiro caso queremos gerar dados no primeiro quadrante e a média da distribuição está localizada no mesmo quadrante. O primeiro quadrante acumula uma probabilidade igual a 0,5162361. No segundo caso a média se localiza no segundo quadrante, fato que pode trazer problemas para gerar valores no primeiro quadrante, que acumula uma probabilidade igual a 0,3714301. O mesmo acontece com o quarto caso, cuja média se localiza no quarto quadrante, assim o primeiro quadrante acumula uma probabilidade igual a 0,3653107. O terceiro caso representa o caso mais difícil pra gerar pontos no primeiro quadrante, pois o mesmo representa uma região de cauda, com baixa probabilidade acumulada, probabilidade essa que é igual a 0,2993847.

As figuras B.1, B.2, B.3 e B.4 apresentam uma comparação gráfica entre os três algoritmos para cada um dos casos especificados acima. Em todos esses gráficos vemos que o CFTP gera valores que se comportam de forma semelhante aos dados gerados pelos outros métodos. Vemos também uma pequena concentração de pontos acima da linha que indica o acúmulo de $95 \%$ de probabilidade, o que é esperado.

\subsubsection{Análise marginal}

Para ter uma melhor idéia do ajuste dos dados simulados a distribuição alvo, exibimos aqui os gráficos dos histogramas das distribuições marginais estimadas. Novamente apresentaremos apenas uma comparação gráfica para cada caso. Através das figuras B.5, B.6, B.7 e B.8, novamente, percebemos que os três métodos geram valores que se comportam de forma semelhante e se adequam ao modelo proposto, normal bivariada truncada, através das distribuições marginais.

Pelos gráficos podemos dizer que para a distribuição normal bivariada truncada, ambos os métodos geraram valores com o mesmo comportamento e portanto os três métodos são eficientes para gerar valores da distribuição normal bivariada truncada no quadrante positivo.

\subsection{Análise do tempo computacional}

Nesta seção apresentamos os tempos, em segundos, que cada um dos três algoritmos leva para gerar uma amostra de tamanho 1000, lembramos que não podemos determinar qual dos três métodos 
é mais rápido, pois a velocidade do amostrador de Gibbs e do CFTP dependem de argumentos escolhidos, arbitrariamente, pelo usuário. A velocidade do Amostrador de Gibbs depende do tamanho do período de aquecimento e do tamanho do espaçamento entre as observações, quanto maiores esses tamanhos mais tempo leva para gerar amostras. Já a velocidade do CFTP depende da escolha do estado $\hat{0}$ quanto mais distante do estado $\hat{1}$ mais tempo leva para as duas cadeias coalescerem. A velocidade do CFTP também depende da sequência de variáveis aleatórias uniformes. Por esses motivos apresentados, não podemos afirmar que um método é mais rápido que outro em definitivo, podemos apenas fazer um estudo de casos, uma comparação para os tempos que os algoritmos levaram para gerar as amostras das quatro distribuições já exibidas acima.

Na tabela 4.1 apresentamos os tempos computacionais gastos pelos três algoritmos para gerar amostras de tamanho 1000, para cada caso especificado, com os parâmetros $\Sigma, \mu_{1}, \mu_{2}, \mu_{3}$ e $\mu_{4}$ todos especificados antes dos truncamentos das distribuições. Os tempos registrados nas colunas referentes ao CFTP foram obtidos somando os tempos que o algoritmo gastou para gerar cada uma das amostras individualmente, pois o CFTP retorna apenas uma observação a cada vez que é executado. Informamos que os tempos registrados na tabela 4.1 foram arrendondados para duas casas decimais.

Percebemos que, no caso $1 \mathrm{com}$ as distribuições truncadas no quadrante positivo, o CFTP foi o mais lento para gerar amostras de todas as distribuições, seguido pelo amostrador de Gibbs e sendo o método de rejeição o mais rápido, como era esperado. Vemos também que o CFTP é mais lento quando o vetor de médias originais se localizam fora da região truncada, quanto mais longe da região de truncamento, menor a probabilidade acumulada dessa região e mais tempo é gasto para gerar pontos dentro dessa região, casos 2, 3, e 4. Com os dados da tabela 4.1, percebemos que gerar pontos dentro do primeiro quadrante quando o vetor de médias está localizado no terceiro quadrante é o caso mais difícil para gerar valores, já que se trata de gerar pontos dentro de uma região de cauda.

Na figura B.9 apresentamos os tempos $T$, tais que a coalescência entre as cadeias de Markov que representam os estados $\hat{0}$ e $\hat{1}$ acontece entre um tempo $-T$ e 0 . Percebemos que a distribuição de $T$ não depende da localização do vetor de médias posteriores ao truncamento, como era esperado, já que o teorema 2.1.1 afirma que o tempo $T$ depende somente de $U_{1}, U_{2}, \ldots$

\subsubsection{Influência da correlação no tempo computacional do CFTP}

Além de comparar a velocidade do CFTP com os outros métodos, estamos interessados em avaliar o efeito da correlação entre as variáveis estudadas. Correlação calculada na distribuição anterior ao truncamento. A partir da matriz de variância e covariância $\Sigma$ calculamos que a correlação antes do truncamento de todas as quatro distribuições é $\rho_{X Y}=0.8819171$, ou seja, as variáveis são altamente correlacionadas positivamente. Geramos novas amostras da distribuição normal bivariada no quadrante positivo, vinte amostras de tamanho mil, ambas com vetor de medias, anterior ao truncamento, igual a $\mu_{1}$. Dez dessas amostras provem da distribuição com matriz de variância e covariância, anterior ao trucamento, igual a $\Sigma=\left[\begin{array}{cc}3.5 & 0 \\ 0 & 4.5\end{array}\right]$, gerando assim uma correlação 
Tabela 4.1: Tempos computacionais, em segundos, gasto pelos três métodos para gerar uma amostra de tamanho 1000

\begin{tabular}{|c|c|c|c|c|c|c|c|}
\hline \multicolumn{4}{|c|}{ Caso 1} & \multicolumn{4}{|c|}{ Caso 2} \\
\hline & CFTP & Gibbs & Rejeição & & CFTP & Gibbs & Rejeição \\
\hline Amostra 1 & 32.21 & 8.12 & 0.09 & Amostra 1 & 40.97 & 8.01 & 0.03 \\
\hline Amostra 2 & 33.26 & 7.90 & 0.02 & Amostra 2 & 40.27 & 7.91 & 0.03 \\
\hline Amostra 3 & 32.32 & 7.93 & 0.03 & Amostra 3 & 39.37 & 7.93 & 0.03 \\
\hline Amostra 4 & 33.98 & 8.05 & 0.02 & Amostra 4 & 40.44 & 7.91 & 0.03 \\
\hline Amostra 5 & 33.59 & 7.93 & 0.02 & Amostra 5 & 40.35 & 7.90 & 0.03 \\
\hline Amostra 6 & 32.63 & 8.02 & 0.02 & Amostra 6 & 40.79 & 7.92 & 0.03 \\
\hline Amostra 7 & 32.83 & 7.91 & 0.02 & Amostra 7 & 42.04 & 7.97 & 0.03 \\
\hline Amostra 8 & 34.02 & 7.99 & 0.02 & Amostra 8 & 41.31 & 7.97 & 0.03 \\
\hline Amostra 9 & 33.61 & 8.02 & 0.03 & Amostra 9 & 38.64 & 7.93 & 0.03 \\
\hline Amostra 10 & 33.57 & 7.94 & 0.02 & Amostra 10 & 42.31 & 7.91 & 0.03 \\
\hline \multicolumn{4}{|c|}{ Caso 3} & \multicolumn{4}{|c|}{ Caso 4} \\
\hline Amostra 1 & 183.62 & 7.92 & 0.04 & Amostra 1 & 38.30 & 7.92 & 0.03 \\
\hline Amostra 2 & 68.54 & 7.89 & 0.03 & Amostra 2 & 38.77 & 7.85 & 0.03 \\
\hline Amostra 3 & 70.31 & 7.92 & 0.04 & Amostra 3 & 37.44 & 7.92 & 0.03 \\
\hline Amostra 4 & 89.19 & 7.87 & 0.04 & Amostra 4 & 37.98 & 7.93 & 0.03 \\
\hline Amostra 5 & 68.75 & 7.93 & 0.04 & Amostra 5 & 37.00 & 7.93 & 0.03 \\
\hline Amostra 6 & 66.71 & 7.86 & 0.04 & Amostra 6 & 37.45 & 7.99 & 0.03 \\
\hline Amostra 7 & 60.14 & 8.00 & 0.04 & Amostra 7 & 37.02 & 7.92 & 0.03 \\
\hline Amostra 8 & 62.10 & 7.89 & 0.03 & Amostra 8 & 36.79 & 7.98 & 0.03 \\
\hline Amostra 9 & 61.00 & 7.94 & 0.04 & Amostra 9 & 37.02 & 8.00 & 0.03 \\
\hline Amostra 10 & 136.56 & 8.00 & 0.03 & Amostra 10 & 38.98 & 7.92 & 0.03 \\
\hline
\end{tabular}

$\rho_{X Y}=0$. As outras dez foram geradas da distribuição cuja matriz de variância e covariância, anterior ao truncamento, é $\Sigma=\left[\begin{array}{cc}3.5 & -3.5 \\ -3.5 & 4.5\end{array}\right]$ gerando uma correlação $\rho_{X Y}=-0.8819171$.

De acordo com dados registrados na tabela 4.2, percebemos que a correlação entre variáveis, $\rho_{X Y}$, tem uma influência no desempenho do CFTP, quanto maior a correlação, positiva, menor o tempo gasto para gerar amostras. Uma possível explicação para este fato é a função de transição utilizada. Em seu trabalho Philippe \& Robert usam uma função de transição $\phi$ baseada no algoritmo de aceitação e rejeição em uma caixa no $\mathbb{R}^{2}$. Com variáveis altamente correlacionadas positivamente, a probabilidade de aceitarmos um ponto sorteado, aleatoriamente, como um ponto pertencente ao quadrante positivo e que satisfaça a equação 3.2 é alta, resultando em um menor número de simulações dentro do algoritmo de aceitação e rejeição diminuindo assim o tempo gasto no algoritmo CFTP. O mesmo poderia não aconteceria se a distribuição normal não tivesse sido truncada no quadrante positivo, se o truncamento fosse no quarto ou no segundo quadrante o efeito da correlação positiva não seria o mesmo, nesse caso a correlação positiva deixaria o CFTP mais lento e a correlação negativa o deixaria mais rápido. 
Tabela 4.2: Tempos computacionais, em segundos, gasto pelo CFTP para gerar amostras com diferentes correlações

\begin{tabular}{l|ccc}
\hline \hline & Correlação positiva & Correlação nula & Correlação negativa \\
\hline Amostra 1 & 32.21 & 48.68 & 137.42 \\
Amostra 2 & 33.26 & 47.92 & 138.86 \\
Amostra 3 & 32.32 & 48.66 & 139.43 \\
Amostra 4 & 33.98 & 51.92 & 138.73 \\
Amostra 5 & 33.59 & 49.50 & 136.76 \\
Amostra 6 & 32.63 & 49.50 & 145.13 \\
Amostra 7 & 32.83 & 47.75 & 141.93 \\
Amostra 8 & 34.02 & 49.02 & 142.01 \\
Amostra 9 & 33.61 & 50.34 & 136.06 \\
Amostra 10 & 33.57 & 48.34 & 142.29 \\
\hline \hline
\end{tabular}

\subsubsection{Considerações finais}

No desenvolvimento desse trabalho descrevemos três métodos para gerar amostras de variáveis aleatórias com distribuição normal bivariada truncada no quadrante positivo. Comparamos os valores gerados pelos três métodos, percebemos que os três métodos geram amostras semelhantes. Não conseguiríamos distinguir qual amostra foi gerada por qual método.

Como o algoritmo de rejeição também é um algoritmo de simulação perfeita, e por ser o método mais rápido, ele foi considerado o melhor, para o truncamento no quadrante positivo e para duas dimensões. Concluímos que a proposta de Philippe \& Robert (2003) embora interessante do ponto de vista teórico, não é uma alternativa competitiva com os usuais algoritmos de Gibbs e rejeição. O CFTP deve ser utilizado em modelos mais complexos. 


\section{Apêndice A}

\section{Algoritmo}

A seguir apresentaremos o algoritmo utilizado para gerar as amostras apresentadas no capítulo 4, implementado a partir do pseudo-código apresentado em Philippe \& Robert (2003). Também apresentaremos comentários sobre alguns blocos de comandos, e sugestões computacionais para a implementação desde algoritmo para que o mesmo gere amostras em mais de duas dimensões e para diferentes regiões de truncamento. Lembramos que o Algoritmo foi escrito em R.

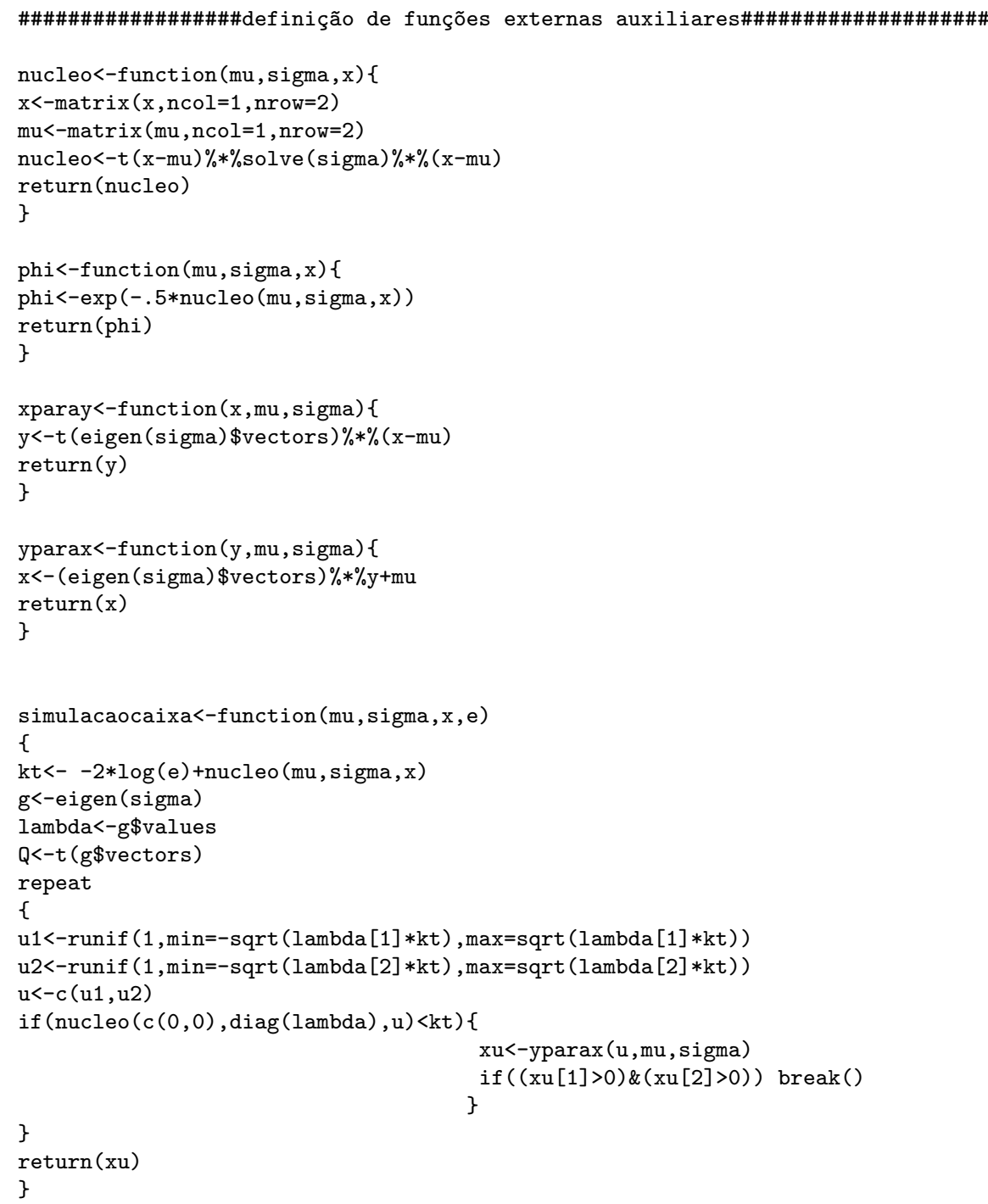




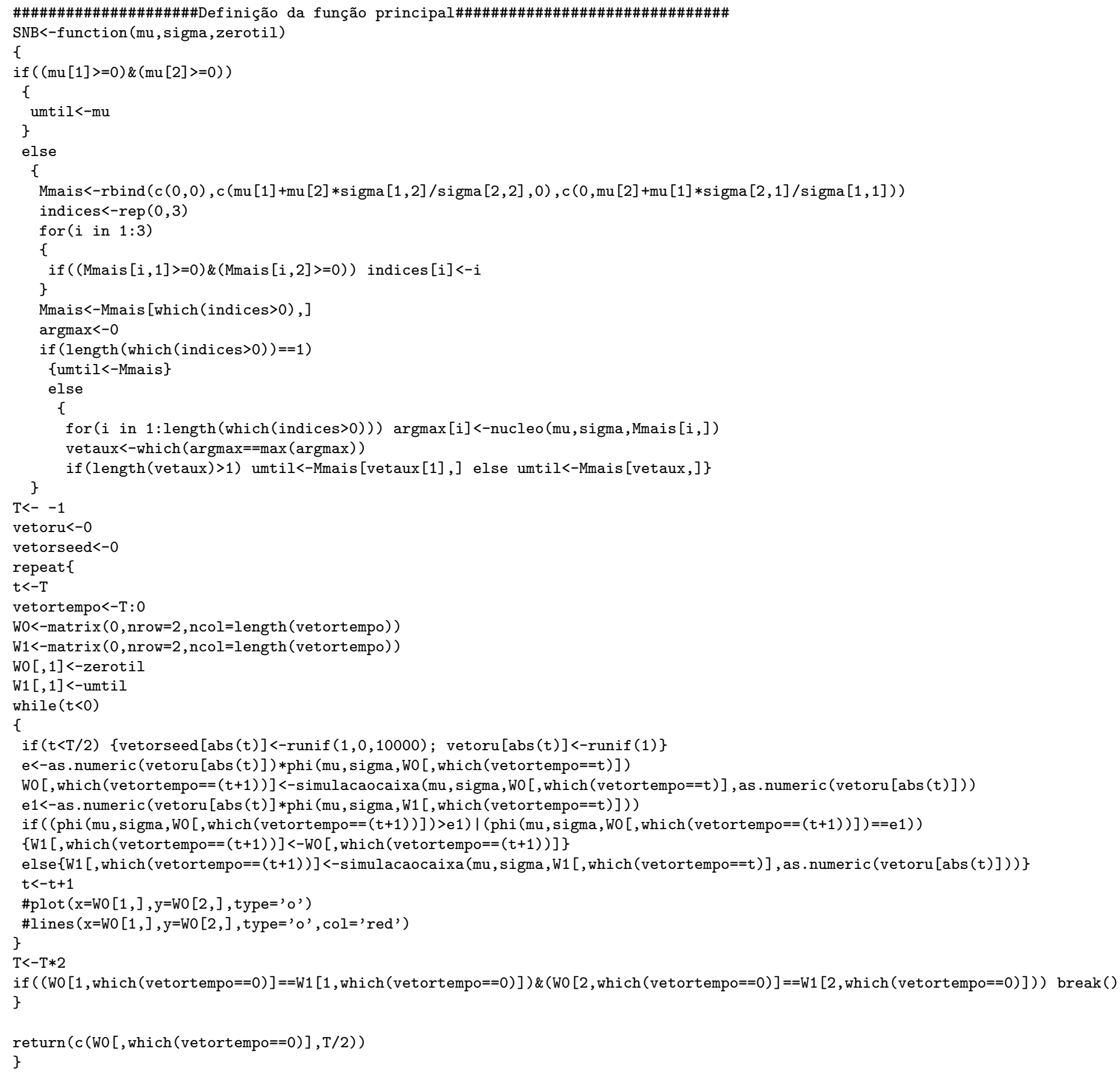

Como o próprio nome diz, as funções auxiliares, são funções definidas para realizar determinados cálculos que serão necessários diversas vezes. Todas essas funções foram desenvolvidas para a manipulação de termos da inequação 3.2, mais precisamente, das formas reduzidas 3.3 e 3.4, lembramos que a transformação de variável utilizada para mudar da equação 3.3 para 3.4 vale para qualquer dimensão maior que dois. As funções denominadas de xparay e yparax fazem a correspondência entre a variável descrita em 3.3 e a variável descrita em 3.4 e vice-versa.

$\mathrm{Na}$ função simulacaocaixa é construída uma caixa que tangencia a região dos pontos que satisfazem 3.2. Nessa caixa simulamos pontos e verificamos se satisfaz ou não 3.2, depois disso verificamos se esse ponto esta ou não no quadrante positivo. Para este procedimento usamos valores 
da variável descrita em 3.4, pois a região de pontos, elipsoide, fica centrada na origem do plano cartesiano, facilitando assim a simulações de pontos para o algoritmo de aceitação e rejeição.

O bloco de comandos a seguir calcula o valor de $\hat{1}$ de acordo com o vetor de médias posteriores ao truncamento, $\mu$, e da matriz de variância e covariância, também posterior ao truncamento, $\Sigma$. Se a media original estiver dentro do conjunto de truncamento então ela irá maximizar a densidade truncada e portanto $\hat{1}=\mu$. Caso contrario $\hat{1}$ poderá estar somente nas bordas do conjunto, podendo inclusive ser a origem do plano cartesiano. O anexo de Philippe \& Robert (2003) mostra em mais detalhes a teoria necessária para determinar $\hat{1}$.

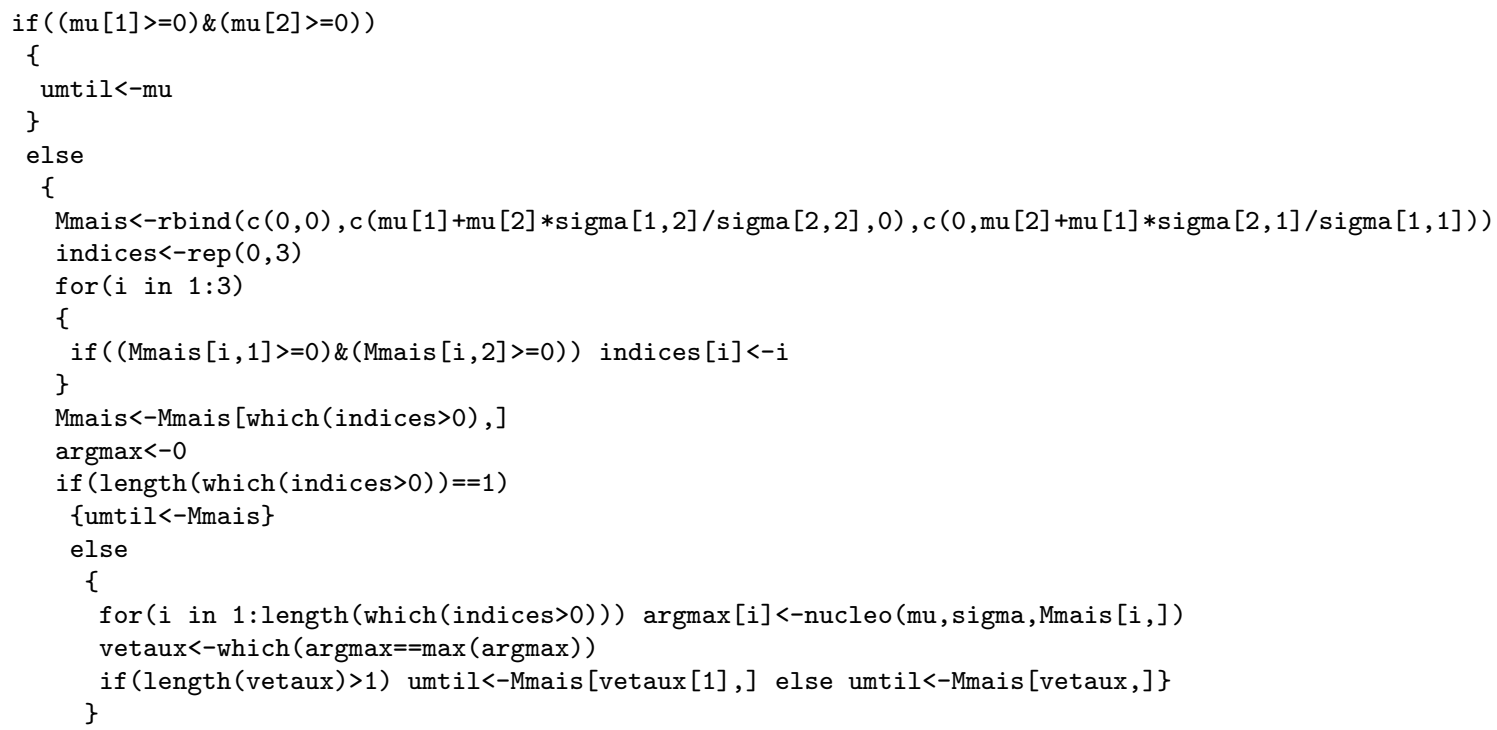

Esse mesmo bloco de funções,mostrado acima, pode ser modificado para calcular $\hat{1}$, quando a variável de interesse é de uma dimensão superior a dois, bastando estipular pontos nas bordas do conjunto de truncamento, utilizando as combinações lineares das compenetres do vetor de médias, $\mu_{1}+\mu_{2} \frac{\sigma_{12}}{\sigma_{22}}$ e $\mu_{2}+\mu_{1} \frac{\sigma_{12}}{\sigma_{11}}$, para determinar os candidatos a $\hat{1}$ no caso do vetor de medias anterior ao truncamento, $\mu$, não pertencer ao conjunto de truncamento.

O grupo de comandos computacionais que deve ser modificado para a implementação do CFTP para dimensões maiores que dois e para outros conjuntos de truncamentos são os comandos presentes

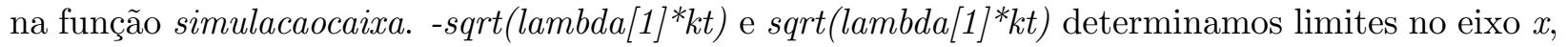
-sqrt(lambda[2]*kt) e $\operatorname{sqrt}($ lambda[2]* $k t$ ) determinamos limites no eixo $y$, portanto os limites para o eixo $i$ da dimensão $i$ são dados por $-\sqrt{\lambda_{i} K_{t}}$ e $\sqrt{\lambda_{i} K_{t}}$. 


\section{Apêndice B}

\section{Gráficos}

Para melhorar a compreensão do leitor, todos os gráficos referenciados no Capítulo 4 foram alocados abaixo
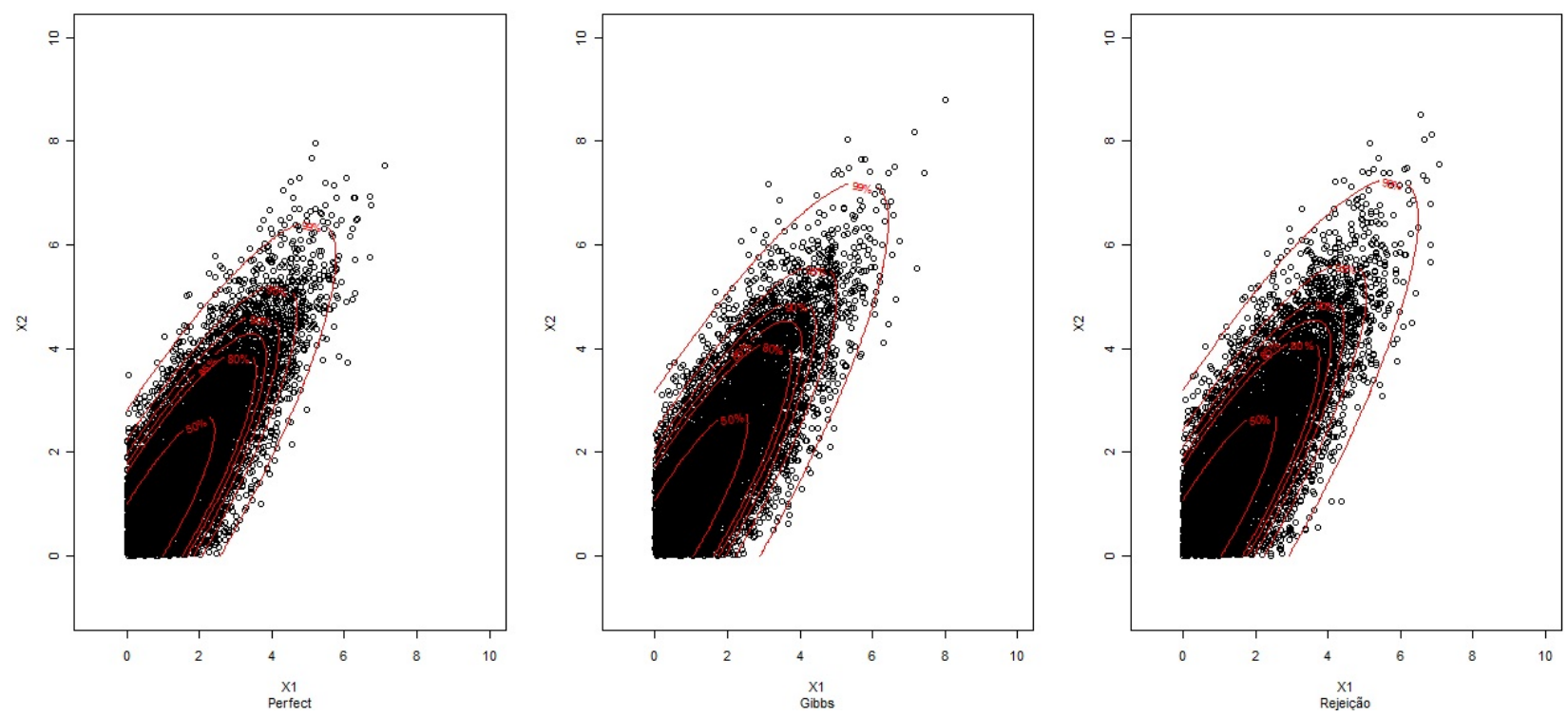

Figura B.1: Amostras da distribuição normal bivariada truncada no quadrante positivo no caso 1, respectivamente, geradas pelos três algoritmos, com a sobreposição de HPDs de $50 \%$ de probabilidade acumulada, $80 \%, 85 \%, 90 \%, 95 \%$ e $99 \%$. 

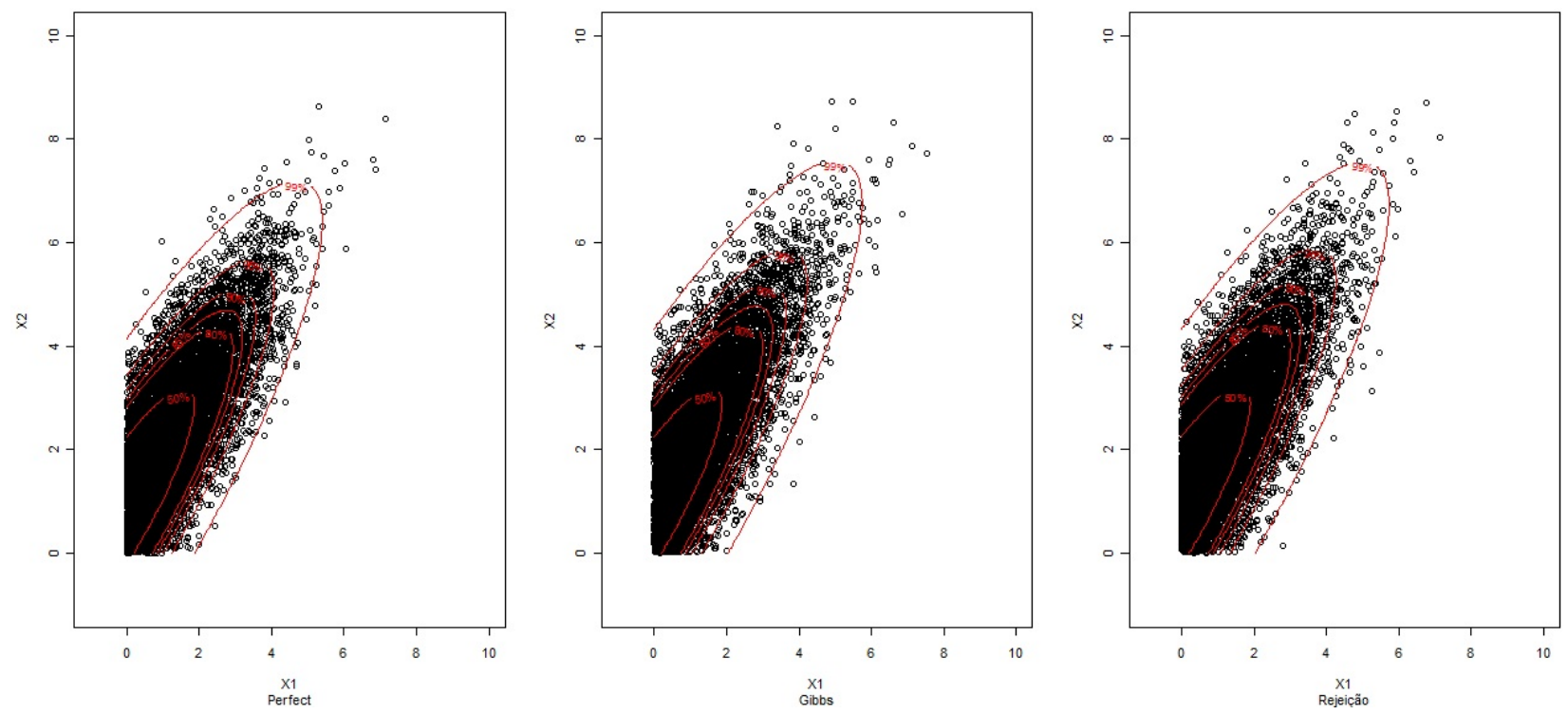

Figura B.2: Amostras da distribuição normal bivariada truncada no quadrante positivo no caso 2, geradas pelos três algoritmos, com a sobreposição de HPDs de $50 \%$ de probabilidade acumulada, $80 \%, 85 \%, 90 \%$, $95 \%$ e $99 \%$.
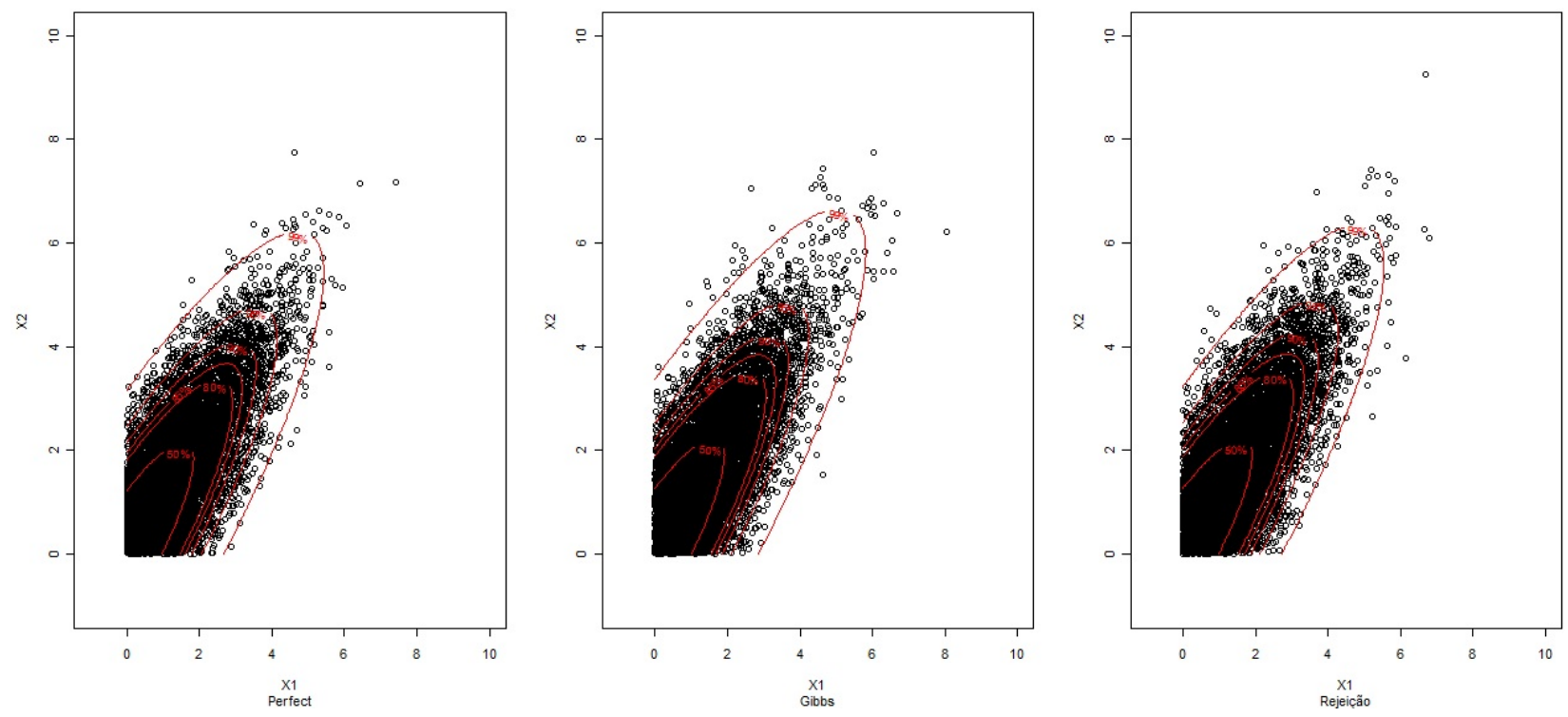

Figura B.3: Amostras da distribuição normal bivariada truncada no quadrante positivo no caso 3, respectivamente, geradas pelos três algoritmos, com a sobreposição de HPDs de $50 \%$ de probabilidade acumulada, $80 \%, 85 \%, 90 \%, 95 \%$ e $99 \%$. 

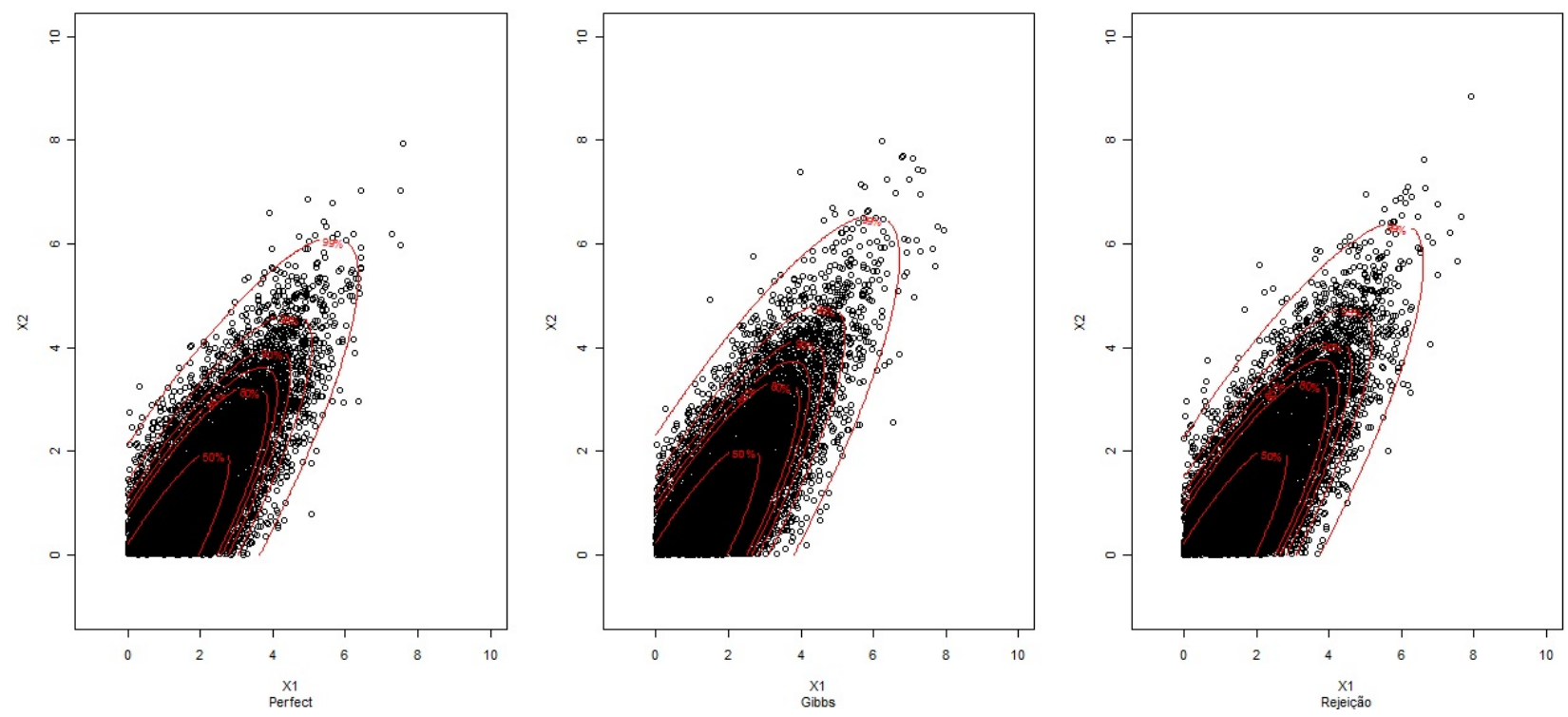

Figura B.4: Amostras da distribuição normal bivariada truncada no quadrante positivo no caso 4, respectivamente, geradas pelos três algoritmos, com a sobreposição de HPDs de $50 \%$ de probabilidade acumulada, $80 \%, 85 \%, 90 \%, 95 \%$ e $99 \%$.
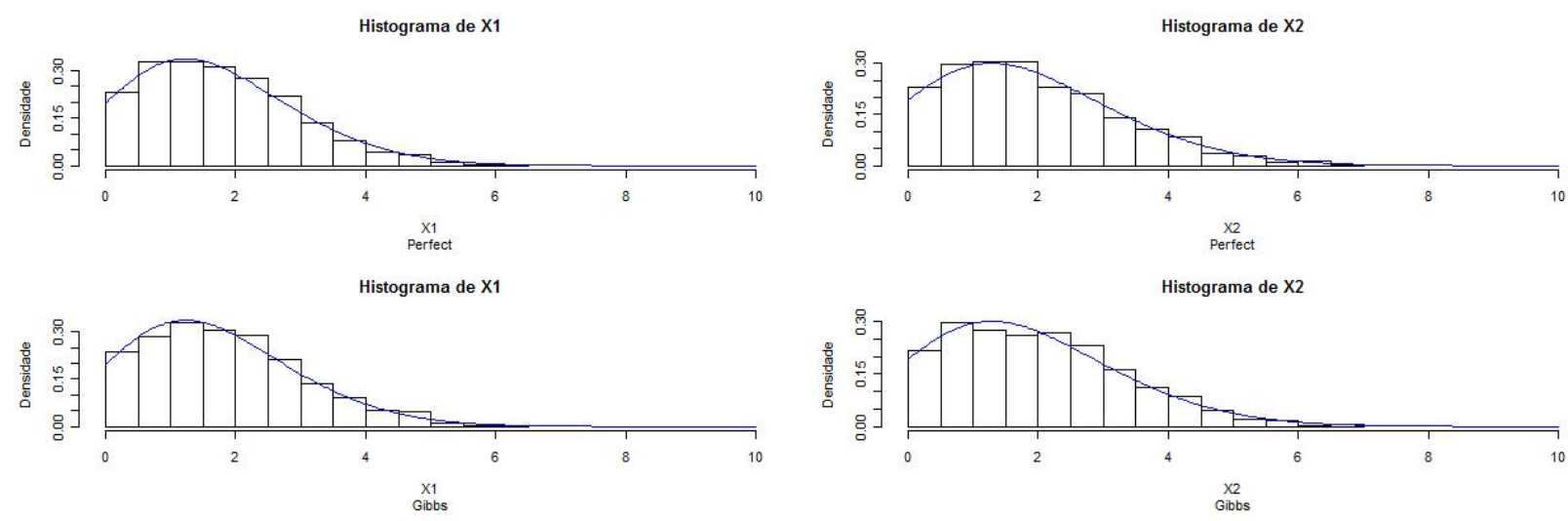

Histograma de X1
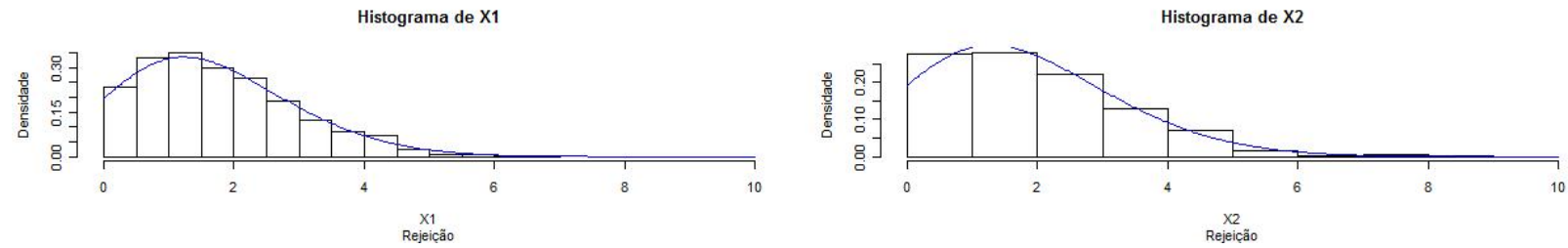

Figura B.5: Histograma das densidades marginais estimadas, com sobreposição da densidade de interesse, para os dados simulados no primeiro caso por cada um dos métodos usados 

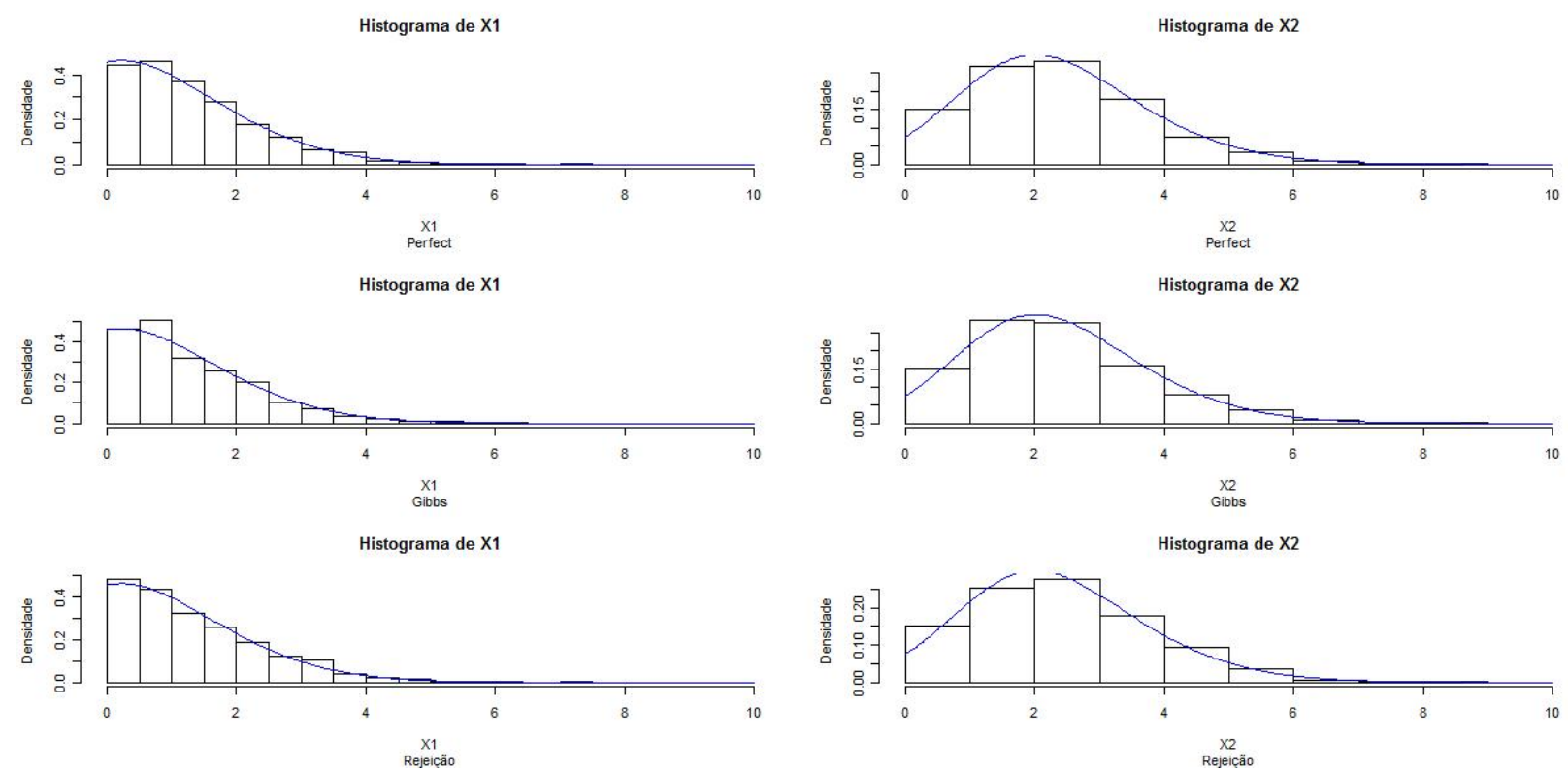

Figura B.6: Histograma das densidades marginais estimadas, com sobreposição da densidade de interesse, para os dados simulados no segundo caso por cada um dos métodos usados
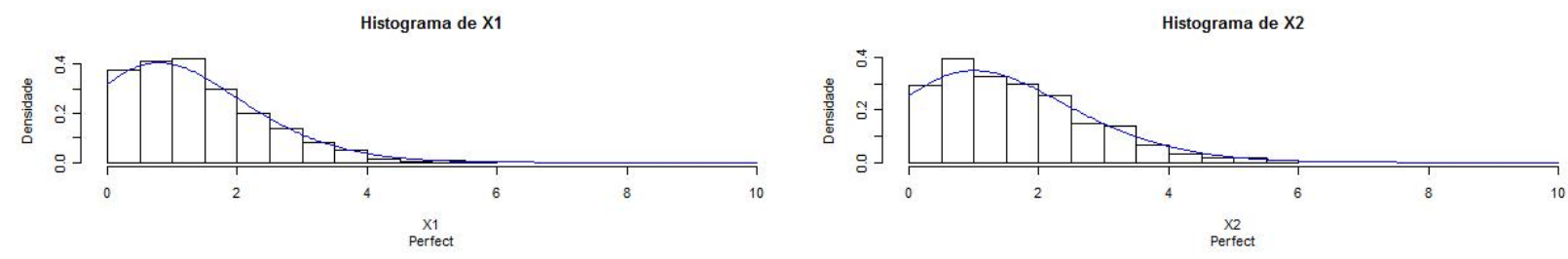

Histograma de X1
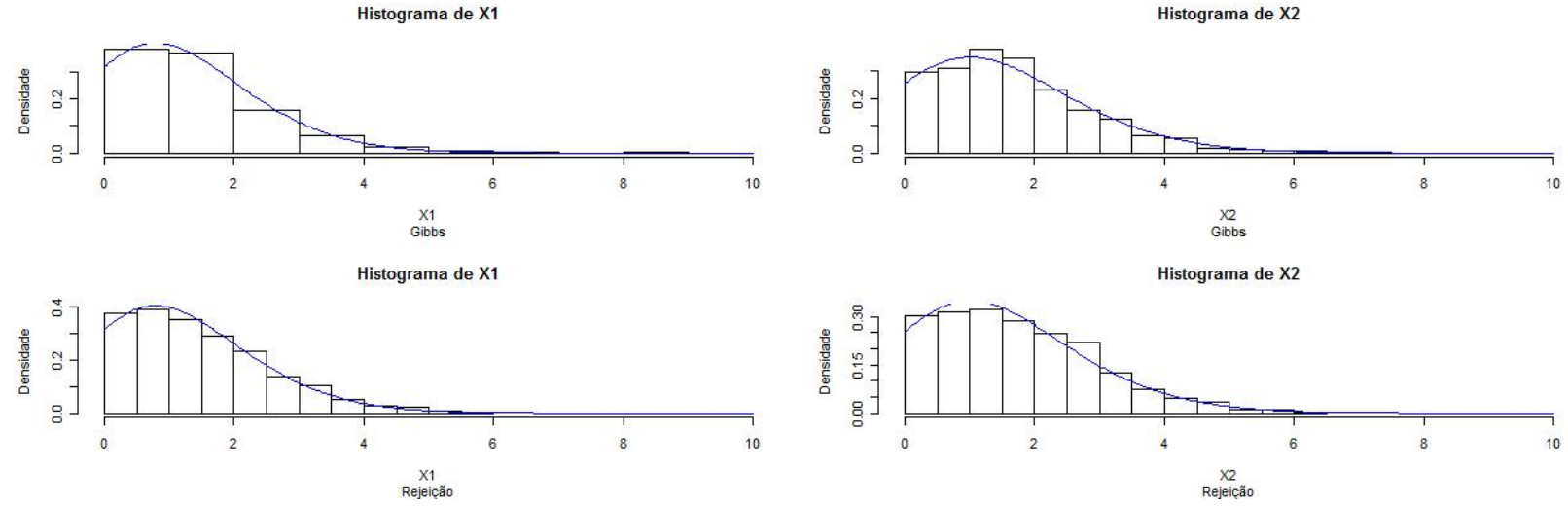

Figura B.7: Histograma das densidades marginais estimadas, com sobreposição da densidade de interesse, para os dados simulados no terceiro caso por cada um dos métodos usados 

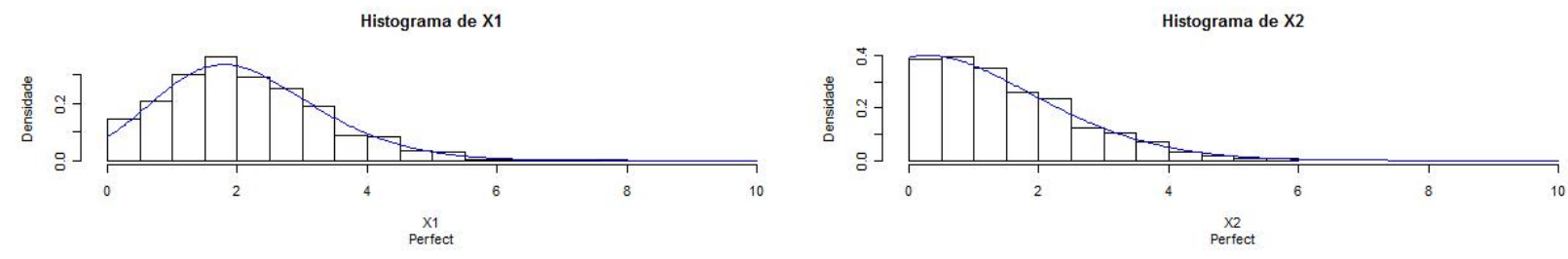

Histograma de X1

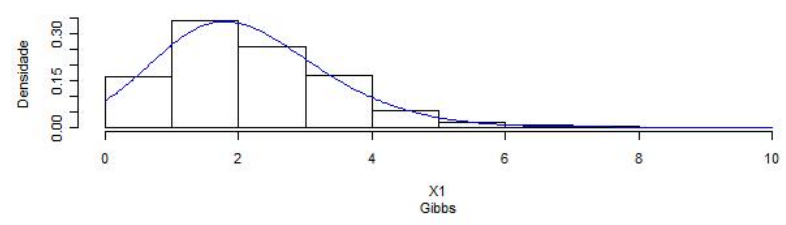

Histograma de X2

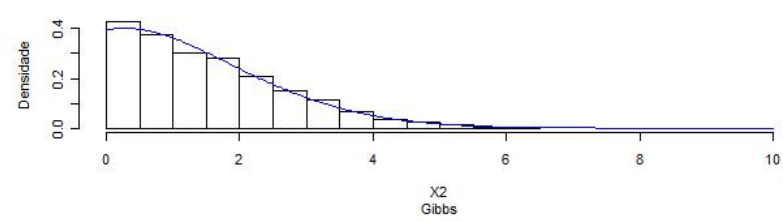

Histograma de X1
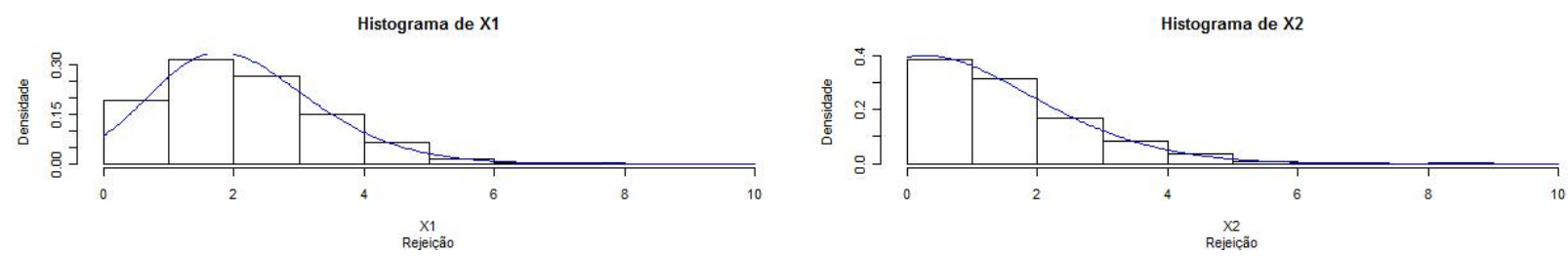

Figura B.8: Histograma das densidades marginais estimadas, com sobreposição da densidade de interesse, para os dados simulados no quarto caso por cada um dos métodos usados
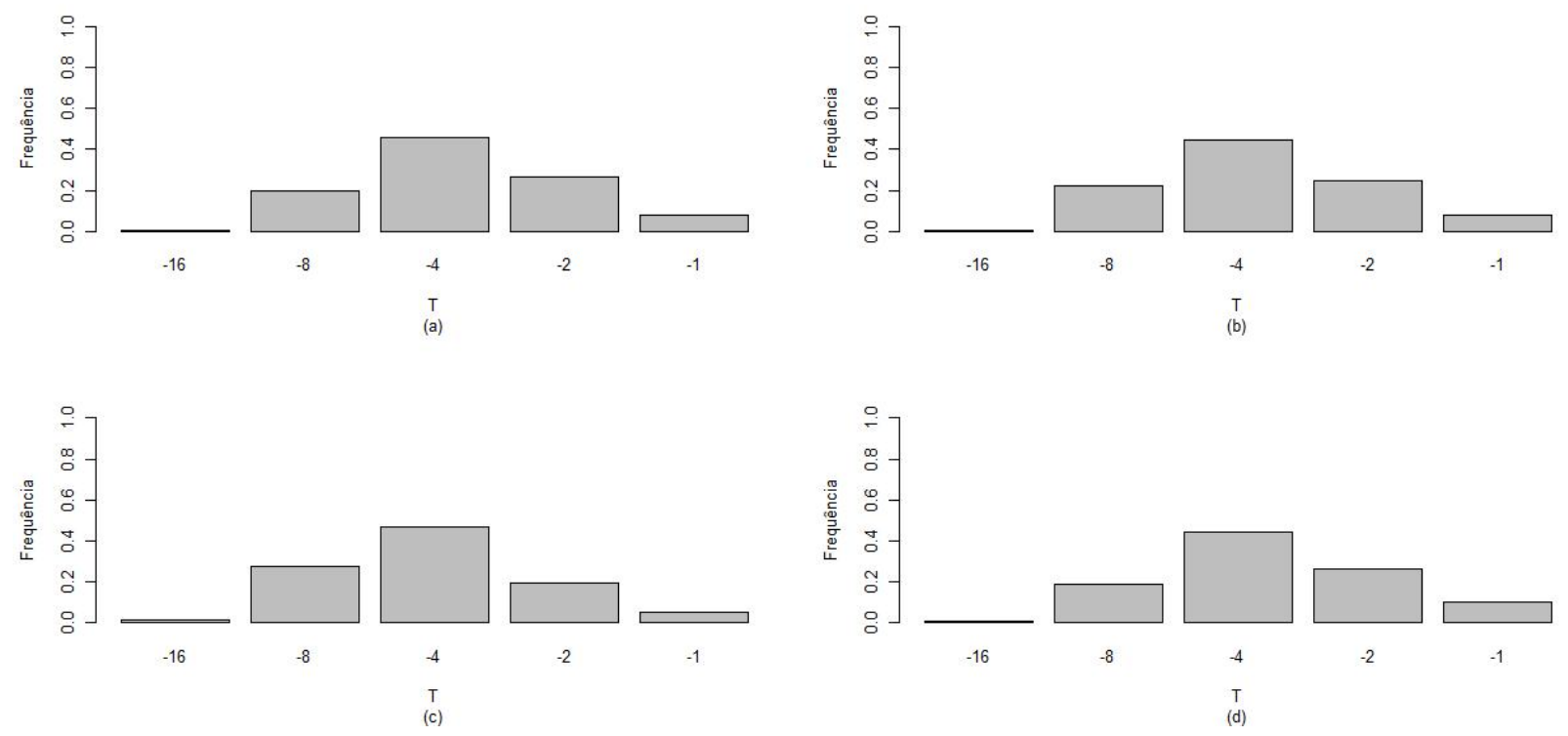

Figura B.9: Histograma dos tempos de retornos para que ocorra a coalescência das cadeias de Markov que representam os estados $\hat{0}$ e $\hat{1}$. O gráfico (a) refere as amostras da distribuição com $\mu_{1}$. O gráfico (b) refere as amostras da distribuição com $\mu_{2}$. O gráfico (c) refere as amostras da distribuição com $\mu_{3}$. O gráfico (d) refere as amostras da distribuição com $\mu_{4}$. 


\section{Referências Bibliográficas}

Casella, G. \& George, E. I. (1992), 'Explaining the gibbs sampler', The American Statistician Association 46(3), 167-174.

Casella, G., Lavine, M. \& Robert, C. P. (2001), 'Explaining the perfect sampler', The American Statistician 55(4), 299-305.

Coelho, E. C. (2001), Simulação perfeita via cadeias de Markov, Dissertação apresentada ao instituto de matemática e estatística da universidade de São Paulo para obtenção do grau de mestre em estatística, São Paulo.

Farias, R. B. A. (2007), Regressão binária bayesiana com o uso de variáveis auxiliares, Dissertação apresentada ao instituto de matemática e estatística da universidade de São Paulo para obtenção do grau de mestre em estatística, São Paulo.

Fernandez, P. J., Ferrari, P. A. \& Grynberg, S. P. (2007), 'Perfectly random sampling of truncated multinormal distributions', Advanced Applied Probability 39, 973-990.

Murdoch, D. \& Green, P. J. (1998), 'Exact sampling from a continous space state', Scandinavian Journal of Statistics 25(3), 483-502.

Philippe, A. \& Robert, C. P. (2003), 'Perfect simulation of positive gaussian distributions', Statistics and Computing 13(2), 179-186.

Propp, J. G. \& Wilson, D. B. (1996), 'Exact sampling with couplesd markov chains and applications to statistical mechanics', Random Structures and Algorithms 9(1-2), 223-252.

Robert, C. (1995), 'Simulation of trauncated normal variables', Statistics and Computing 5(2), 121125.

Ross, S. M. (2007), Introduction to Probability Models, 9th edn, AP, Berkley, California. 